\title{
Synthesis and application of phosphorus dendrimer immobilized azabis(oxazolines)
}

Anja Gissib| $\left.\right|^{a, b}$, Clement Padiée, ${ }^{a, b}$, Markus Hager, ${ }^{a, b}$, Florian Jaroschik ${ }^{a, b}$, Ramesh Rasappan $^{a}$, Erick Cuevas-Yanez ${ }^{\mathrm{a}}$, Cédric-Olivier Turrin ${ }^{\mathrm{b}}$, Anne-Marie Caminade $^{\mathrm{b}}$, Jean-Pierre Majoral| ${ }^{\mathrm{b},{ }^{,}}$, Oliver Reiser ${ }^{\mathrm{a},{ }^{\star}}$

${ }^{a}$ Institut für Organische Chemie, Universität Regensburg, Universitätsstr. 31, 93053 Regensburg, Germany

${ }^{b}$ Laboratoire de Chimie de Coordination, 205 route de Narbonne, 31077 Toulouse cedex 4, France

Oliver.Reiser@chemie.uni-regensburg.de; majoral@1cc-toulouse.fr 


\section{General method for the benzoylation of hydrobenzoine and cyclohexanediol:}

Dendrimer bearing aza-bis(oxazoline) end groups (generation 1: $28 \mathrm{mg}, 4.310^{-2}$ mmol; generation 2: $30 \mathrm{mg}, 2.110^{-3} \mathrm{mmol}$; generation 3: $31 \mathrm{mg}, 1.0510^{-3} \mathrm{mmol}$; each case corresponds to $5 \mathrm{~mol} \%$ end groups) and copper chloride (6 mg, $4.510^{-2}$ $\mathrm{mmol}$ ) in freshly distilled (onto $\mathrm{P}_{4} \mathrm{O}_{10}$ ) dichloromethane $(5 \mathrm{~mL}$ ) were stirred at room temperature in a Schlenk tube for $2 \mathrm{~h} .1 .0 \mathrm{mmol}$ of diol $(214 \mathrm{mg}$ of hydrobenzoine or $117 \mathrm{mg}$ of cyclohexandiol) and $1.0 \mathrm{mmol}$ of DIPEA (165 $\mu \mathrm{L})$ were added to this mixture. The resulting green solution for the first and second generation dendrimers and the green suspension for the third generation were cooled to $0^{\circ} \mathrm{C}$ before adding $0.5 \mathrm{mmol}$ of benzoyl chloride $(57 \mu \mathrm{L})$. The mixture was stirred at $0^{\circ} \mathrm{C}$ for $4 \mathrm{~h}$. The reaction was finished after this time as monitored by TLC with the complete disappearance of benzoyl chloride. Then, $25 \mathrm{~mL}$ of hexanes were added to precipitate the dendritic catalyst, which was recovered by filtration and washed twice with a 1:5 DCM/hexanes mixture. The precipitated catalyst was dried and kept for a future use following the same procedure without reloading with copper. The filtrate and the washing solution were evaporated together to give an oily solid, purified by chromatography onto a silica gel column (eluent: hexanes/ethyl acetate 5:1) to afford the coupling products.

\section{4-tert-Butyl-dimethyl-silyloxy-phenethyl iodide (2):}

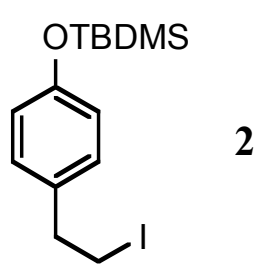

A solution of methanesulfonic acid 2-[4-(tert-butyl-dimethyl-silyloxy)-phenyl]-ethyl ester (2.0 g, $6.06 \mathrm{mmol}, 1$ eq.) and sodium iodide (9.08 g, $60.6 \mathrm{mmol}, 10$ eq.) in 50 $\mathrm{mL}$ acetone was stirred for $24 \mathrm{~h}$ at room temperature in the dark. After evaporation of the solvent, the remaining solid was dissolved in water and dichloromethane, the organic layer was separated and the aqueous layer was extracted twice with dichloromethane. The combined organic layers were dried over $\mathrm{MgSO}_{4}$ and after evaporation of the solvent the crude product was purified by silica gel column 
chromatography (hexanes 100\%). The product 2 was obtained as colourless oil in $93 \%$ yield $(2.04 \mathrm{~g}, 5.64 \mathrm{mmol})$.

${ }^{1} \mathrm{H}-\mathrm{NMR}\left(300 \mathrm{MHz}, \mathrm{CDCl}_{3}\right): \delta=0.18\left(\mathrm{~s}, 6 \mathrm{H}, \mathrm{Si}\left(\mathrm{CH}_{3}\right)_{2}\right), 0.97\left(\mathrm{~s}, 9 \mathrm{H}, \mathrm{SiC}\left(\mathrm{CH}_{3}\right)_{3}\right), 3.09$ (t, $\left.2 \mathrm{H}, J=8.3, \mathrm{CH}_{2} \mathrm{CH}_{2} \mathrm{l}\right), 3.30\left(\mathrm{~m}, 2 \mathrm{H}, \mathrm{CH}_{2} \mathrm{CH}_{2} \mathrm{l}\right), 6.74-6.79\left(\mathrm{~m}, 2 \mathrm{H}, \mathrm{CH}_{\text {aromatic }}\right)$, 7.01-7.06 (m, $\left.2 \mathrm{H}, \mathrm{CH}_{\text {aromatic }}\right)$.

\section{\{2-[4-(tert-Butyl-dimethyl-silanyloxy)-phenyl]-ethyl\}-bis-(4-isopropyl-4,5-} dihydro-oxazol-2-yl)-amine (3):

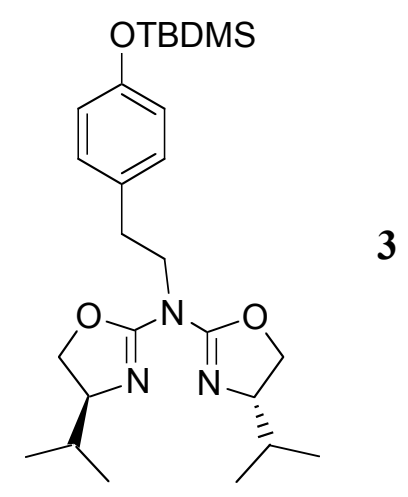

A solution of aza-bis(oxazoline) 1 ( $0.239 \mathrm{~g}, 1.0 \mathrm{mmol}, 1.0 \mathrm{eq}$.) in $4 \mathrm{~mL}$ dry THF was cooled to $-78^{\circ} \mathrm{C}$ and a $1.6 \mathrm{~N}$ solution of $\mathrm{n}$-BuLi $(0.68 \mathrm{~mL}, 1.1 \mathrm{mmol} 1.1$ eq.) was added and left under stirring for $10 \mathrm{~min}$. Then a solution of linker $2(0.72 \mathrm{~g}, 2.0 \mathrm{mmol}$, 2.0 eq.) in $4 \mathrm{~mL}$ THF was added and the reaction mixture was warmed to $28^{\circ} \mathrm{C}$ and left under stirring for $40 \mathrm{~h}$. The solvent was evaporated and the crude mixture was taken up in $10 \mathrm{~mL}$ dichloromethane and $10 \mathrm{~mL}$ saturated $\mathrm{NaHCO}_{3}$ solution. The organic layer was separated and the aqueous layer was extracted twice with $10 \mathrm{~mL}$ dichloromethane. The combined organic layers were dried over $\mathrm{MgSO}_{4}$, the solvent was removed and the product was purified by column chromatography on basic alumina (hexanes/ethyl acetate $=85 / 15$ ). The product 3 was obtained as colourless crystals in $78 \%$ yield $(0.369 \mathrm{~g}, 0.78 \mathrm{mmol})$.

${ }^{1} \mathrm{H}-\mathrm{NMR}\left(250 \mathrm{MHz}, \mathrm{CDCl}_{3}\right): \delta=0.16\left(\mathrm{~s}, 6 \mathrm{H}, \mathrm{Si}\left(\mathrm{CH}_{3}\right)_{2}\right), 0.86(\mathrm{~d}, 6 \mathrm{H}, J=6.8$, $\left.\mathrm{CH}\left(\mathrm{CH}_{3}\right)_{2}\right), 0.95\left(\mathrm{~d}, 6 \mathrm{H}, J=6.8, \mathrm{CH}\left(\mathrm{CH}_{3}\right)_{2}\right), 0.95\left(\mathrm{~s}, 9 \mathrm{H}, \mathrm{SiC}\left(\mathrm{CH}_{3}\right)_{3}\right), 1.70(\mathrm{~m}, 2 \mathrm{H}$, $\left.\mathrm{CH}\left(\mathrm{CH}_{3}\right)_{2}\right), 2.92\left(\mathrm{~m}, 2 \mathrm{H}, \mathrm{CH}_{2} \mathrm{CH}_{2} \mathrm{~N}\right), 3.83\left(\mathrm{~m}, 2 \mathrm{H}, \mathrm{CHCH}_{2}\right), 3.99\left(\mathrm{~m}, 2 \mathrm{H}, \mathrm{NCH}_{2}\right)$, 
$4.08\left(\mathrm{dd}, 2 \mathrm{H}, J=8.3,7.0, \mathrm{CH}_{2} \mathrm{CH}\right.$ ), $4.34\left(\mathrm{dd}, 2 \mathrm{H}, J=8.3,9.0, \mathrm{CH}_{2} \mathrm{CH}\right), 6.70-6.76$

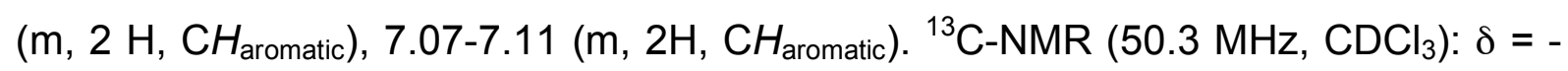
$4.4\left(\mathrm{~s}, \mathrm{Si}\left(\mathrm{CH}_{3}\right)_{2}\right), 17.8\left(\mathrm{~s}, \mathrm{CH}\left(\mathrm{CH}_{3}\right)_{2}\right), 18.1\left(\mathrm{~s}, \mathrm{SiC}\left(\mathrm{CH}_{3}\right)_{3}\right), 18.7\left(\mathrm{~s}, \mathrm{CH}\left(\mathrm{CH}_{3}\right)_{2}\right), 25.6$ (s, $\left.\mathrm{SiC}\left(\mathrm{CH}_{3}\right)_{3}\right), 32.9\left(\mathrm{~s}, \mathrm{CH}\left(\mathrm{CH}_{3}\right)_{2}\right), 33.4\left(\mathrm{~s}, \mathrm{CH}_{2} \mathrm{CH}_{2} \mathrm{~N}\right), 51.5\left(\mathrm{~s}, \mathrm{CH}_{2} \mathrm{CH}_{2} \mathrm{~N}\right), 69.8$ (s, $\mathrm{CHCH}_{2}$ ), 71.2 (s, $\mathrm{CHCH}_{2}$ ), 119.8 (s, C(ortho)), 129.9 (s, $\mathrm{C}$ (meta)), 131.5 (s, C(para)), 153.9 (s, C(ipso)), 156.9 (s, OCN). MS (PI-EIMS, 70eV) $\mathrm{m} / \mathrm{z}(\%)=73.1$ (16.8), 177.1 (91.6), 178.2 (27.4), 196.2 (61.9), 234.2 (100), 235.2 (38.0), 473.4 (12.3). HRMS $\left(\mathrm{C}_{26} \mathrm{H}_{43} \mathrm{~N}_{3} \mathrm{O}_{3} \mathrm{Si}\right)$ calculated: 473.3074 , found: 473.3075 .

\section{4-\{2-[Bis-(4-isopropyl-4,5-dihydro-oxazol-2-yl)-amino]-ethyl\}-phenol (4):}

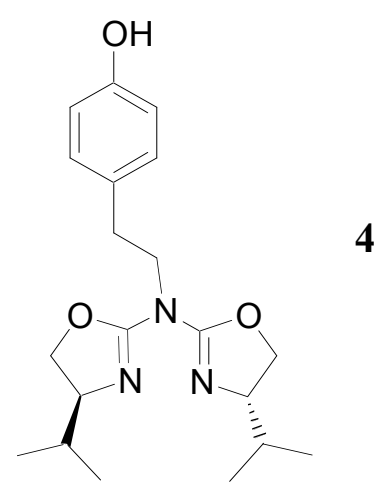

A mixture of linker-bound aza-bis(oxazoline) $3(0.355 \mathrm{~g}, 0.75 \mathrm{mmol}, 1.0$ eq.) and $\mathrm{KOH}(0.084 \mathrm{~g}, 1.50 \mathrm{mmol}, 2.0 \mathrm{eq}$.) in ethanol $(4 \mathrm{~mL})$ was stirred at room temperature until the reaction was finished as indicated by TLC. The solvent was evaporated and the crude material was taken up in dichloromethane and washed twice with water. The combined water layers were then extracted three times with dichloromethane. The organic layers were combined and dried with $\mathrm{MgSO}_{4}$. Evaporation of the solvent gave the product 4 as colourless oil in $90 \%$ yield $(0.242 \mathrm{~g}, 0.68 \mathrm{mmol})$.

${ }^{1} \mathrm{H}-\mathrm{NMR}\left(200 \mathrm{MHz}, \mathrm{CDCl}_{3}\right): \delta=0.85\left(\mathrm{~d}, 6 \mathrm{H}, \mathrm{J}=6.3, \mathrm{CH}\left(\mathrm{CH}_{3}\right)_{2}\right), 0.93(\mathrm{~d}, 6 \mathrm{H}, J=6.3$, $\left.\mathrm{CH}\left(\mathrm{CH}_{3}\right)_{2}\right), 1.70-1.81\left(\mathrm{~m}, 2 \mathrm{H}, \mathrm{CH}\left(\mathrm{CH}_{3}\right)_{2}\right), 2.90\left(\mathrm{~m}, 2 \mathrm{H}, \mathrm{CH}_{2} \mathrm{CH}_{2} \mathrm{~N}\right), 3.84-3.91(\mathrm{~m}, 2$ $\mathrm{H}, \mathrm{CHCH}_{2}$ ), 3.94-4.01 (m, $2 \mathrm{H}, \mathrm{NCH}_{2}$ ), 4.09 (dd, $2 \mathrm{H}, \mathrm{J}=8.3,7.0, \mathrm{CH}_{2} \mathrm{CH}$ ), 4.28 (dd, $\left.2 \mathrm{H}, J=8.3,9.0, \mathrm{CH}_{2} \mathrm{CH}\right), 6.70-6.73\left(\mathrm{~m}, 2 \mathrm{H}, \mathrm{CH}_{\text {aromatic }}\right), 7.03-7.06(\mathrm{~m}, 2 \mathrm{H}$, $\left.\mathrm{CH}_{\text {aromatic }}\right) .{ }^{13} \mathrm{C}-\mathrm{NMR}\left(50.3 \mathrm{MHz}, \mathrm{CDCl}_{3}\right): \delta=17.9\left(\mathrm{~s}, \mathrm{CH}\left(\mathrm{CH}_{3}\right)_{2}\right), 19.0\left(\mathrm{~s}, \mathrm{CH}\left(\mathrm{CH}_{3}\right)_{2}\right)$, $32.9\left(\mathrm{~s}, \mathrm{CH}\left(\mathrm{CH}_{3}\right)_{2}\right), 34.0\left(\mathrm{~s}, \mathrm{CH}_{2} \mathrm{CH}_{2} \mathrm{~N}\right), 51.7\left(\mathrm{~s}, \mathrm{CH}_{2} \mathrm{CH}_{2} \mathrm{~N}\right), 69.8\left(\mathrm{~s}, \mathrm{CHCH}_{2}\right), 71.1$ (s, $\mathrm{CHCH}_{2}$ ), 115.9 (s, C(ortho)), 129.4 (s, C(para)), 130.4 (s, C(meta)), 156.2 (s, 
C(ipso)), 157.7 (s, OCN). MS (Cl-MS, $\left.\mathrm{NH}_{3}\right) \mathrm{m} / \mathrm{z}(\%)=130.1$ (5.4), $147.1(24.8), 249.1$ (38.1), 250.2 (5.7), $360.2\left(4.7, \mathrm{MH}^{+}\right)$.

General procedure for the coupling of linker-bound aza-bis(oxazoline) 4 to dendrimers of the first, second and third generation:

To a solution of linker-bound aza-bis(oxazoline) 4 (1.2 eq. per $\mathrm{Cl}$ on dendrimer) in 5 $\mathrm{mL}$ dry $\mathrm{THF}$, the dendrimer (1 eq.) and cesium carbonate $(2.5$ eq. per $\mathrm{Cl}$ on dendrimer) were added and the reaction was stirred at room temperature until ${ }^{31} \mathrm{P}$ NMR indicated that the reaction was complete, usually $24 \mathrm{~h}$. After centrifugation the cesium salts were filtered off and the filtrate was concentrated. Then the dendrimer was precipitated with pentane at $0^{\circ} \mathrm{C}$ and the solution was filtered via cannula. This washing step with $\mathrm{THF} /$ pentane was repeated until all the excess aza-bis(oxazoline) 4 was removed. The pure dendrimer was dried under vacuum which resulted in foaming of the product.

Dodekakis-(4-\{2-[bis-(4-isopropyl-4,5-dihydro-oxazol-2-yl)-amino]-ethyl\}phenoxy)-cyclotriphosphazene $\left(\mathbf{G}_{1} \bullet 4\right)$ :

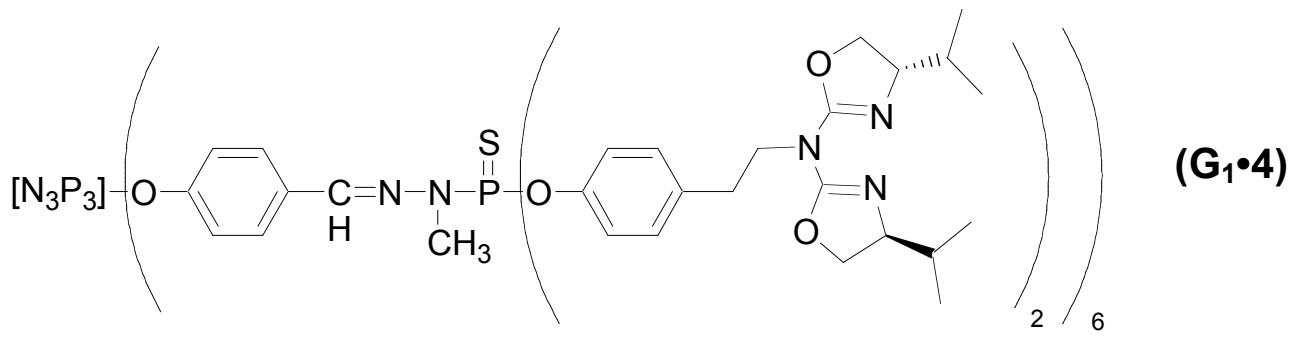

According to the general procedure, linker-bound aza-bis(oxazoline) 4 (0.15 g, 0.42 $\mathrm{mmol}), \mathrm{Cs}_{2} \mathrm{CO}_{3}(0.31 \mathrm{~g}, 0.95 \mathrm{mmol})$ and the dendrimer $\mathbf{G}_{1}(0.058 \mathrm{~g}, 0.032 \mathrm{mmol})$ were reacted to give the product $\mathbf{G}_{1} \bullet \mathbf{4}$ as a white solid in $84 \%$ yield $(0.153 \mathrm{~g}, 0.027$ $\mathrm{mmol})$.

$\left.{ }^{31} \mathrm{P}-\mathrm{NMR}\{1 \mathrm{H}\}(81 \mathrm{MHz}, \mathrm{CDCl})_{3}\right): \delta=11.7\left(\mathrm{~s}, \mathrm{P}_{0}\right), 66.5\left(\mathrm{~s}, \mathrm{P}_{1}\right)$.

${ }^{1} \mathrm{H}-\mathrm{NMR}\left(200 \mathrm{MHz}, \mathrm{CDCl}_{3}\right): \delta=0.80\left(\mathrm{~d}, 72 \mathrm{H}, J=6.8, \mathrm{CH}\left(\mathrm{CH}_{3}\right)_{2}\right), 0.90(\mathrm{~d}, 72 \mathrm{H}, J=$ 6.8, $\left.\mathrm{CH}\left(\mathrm{CH}_{3}\right)_{2}\right), 1.55-1.77\left(\mathrm{~m}, 24 \mathrm{H}, \mathrm{CH}\left(\mathrm{CH}_{3}\right)_{2}\right), 2.90-2.95\left(\mathrm{~m}, 24 \mathrm{H}, \mathrm{CH}_{2} \mathrm{CH}_{2} \mathrm{~N}\right)$, 3.19$3.24\left(\mathrm{~d}, 18 \mathrm{H}, J=11, \mathrm{P}_{1} \mathrm{NCH}_{3}\right), 3.68-3.83\left(\mathrm{~m}, 24 \mathrm{H}, \mathrm{CHCH}_{2}\right), 3.92-4.05(\mathrm{~m}, 48 \mathrm{H}$, 
$\mathrm{NCH}_{2}$ and $\left.\mathrm{CH}_{2} \mathrm{CH}\right), 4.26\left(\mathrm{dd}, 24 \mathrm{H}, J=8.3,9.0, \mathrm{CH}_{2} \mathrm{CH}\right), 6.99-7.17(\mathrm{~m}, 58 \mathrm{H}$, $\mathrm{CH}_{\text {aromatic }}$ ), 7.58-7.62 (m, $\left.12 \mathrm{H}, \mathrm{CH}_{\text {aromatic }}\right), 7.58(\mathrm{~s}, 6 \mathrm{H}, \mathrm{CHN}) .{ }^{13} \mathrm{C}-\mathrm{NMR}(50.3 \mathrm{MHz}$, $\left.\mathrm{CDCl}_{3}\right): \delta=17.8\left(\mathrm{~s}, \mathrm{CH}\left(\mathrm{CH}_{3}\right)_{2}\right), 18.7\left(\mathrm{~s}, \mathrm{CH}\left(\mathrm{CH}_{3}\right)_{2}\right), 32.8\left(\mathrm{~s}, \mathrm{CH}\left(\mathrm{CH}_{3}\right)_{2}\right.$ and $\left.\mathrm{NCH}_{3}\right)$, 33.6 (s, $\mathrm{CH}_{2} \mathrm{CH}_{2} \mathrm{~N}$ ), 51.0 (s, $\mathrm{CH}_{2} \mathrm{CH}_{2} \mathrm{~N}$ ), 69.7 (s, $\mathrm{CHCH}_{2}$ ), 71.1 (s, $\left.\mathrm{CHCH}_{2}\right), 121.1$ (s, $C_{0}$ (ortho) and $C_{1}$ (ortho)), 128.2 (s, $C_{0}$ (meta)), 130.2 (s, $C_{1}$ (meta)), 132.1 (s, $C_{0}$ (para)) $136.1\left(\mathrm{~s}, C_{1}(\right.$ para $\left.)\right), 138.7\left(\mathrm{~d},{ }^{3} J_{\mathrm{CP}}=14, C \mathrm{HN}\right), 149.0\left(\mathrm{~s}, C_{0}\right.$ (ipso)), $151.2\left(\mathrm{~d},{ }^{2} J_{\mathrm{CP}}=\right.$ 7.0, $C_{1}$ (ipso)), 156.7 (OCN).

Eikosa-tetrakis-(4-\{2-[bis-(4-isopropyl-4,5-dihydro-oxazol-2-yl)-amino]-ethyl\}phenoxy)-cyclotriphosphazene $\left(G_{2} \bullet 4\right)$ :

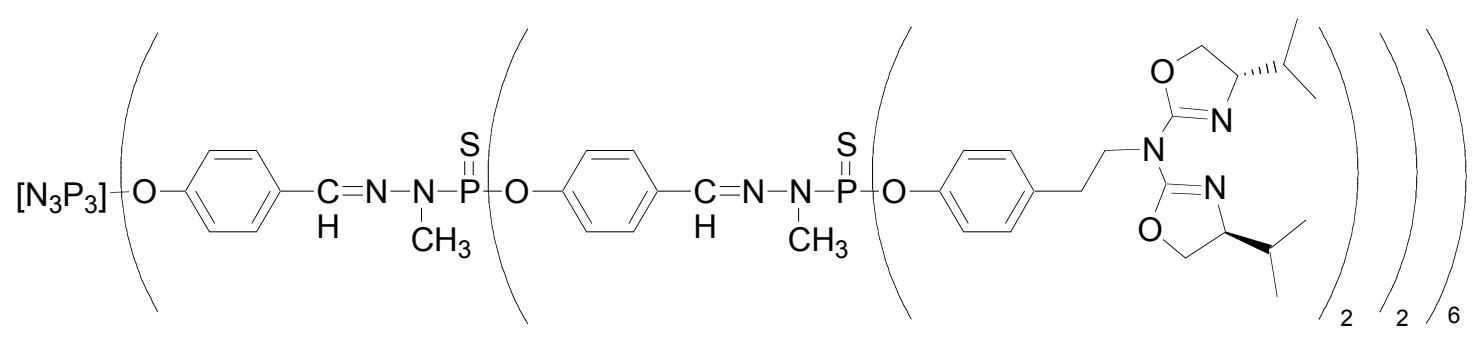

$\mathbf{G}_{2} \bullet 4$

According to the general procedure, linker-bound aza-bis(oxazoline) 4 (0.25 g, 0.696 $\mathrm{mmol}), \mathrm{Cs}_{2} \mathrm{CO}_{3}(0.47 \mathrm{~g}, 1.45 \mathrm{mmol})$ and the dendrimer $\mathbf{G}_{2}(0.116 \mathrm{~g}, 0.024 \mathrm{mmol})$ were reacted to give the product $\mathbf{G}_{2} \cdot \mathbf{4}$ as a white solid in $82 \%$ yield $(0.25 \mathrm{~g}, 0.02$ $\mathrm{mmol})$.

${ }^{31} \mathrm{P}-\mathrm{NMR}\{1 \mathrm{H}\}\left(81 \mathrm{MHz}, \mathrm{CDCl}_{3}\right): \delta=11.7$ (s, $\left.\mathrm{P}_{0}\right), 66.1$ (s, $\left.\mathrm{P}_{1}\right), 66.5$ (s, $\left.\mathrm{P}_{2}\right)$.

${ }^{1} \mathrm{H}-\mathrm{NMR}\left(200 \mathrm{MHz}, \mathrm{CDCl}_{3}\right): \delta=0.80\left(\mathrm{~d}, 144 \mathrm{H}, J=6.2, \mathrm{CH}\left(\mathrm{CH}_{3}\right)_{2}\right), 0.88(\mathrm{~d}, 144 \mathrm{H}, J$ $\left.=6.2, \mathrm{CH}\left(\mathrm{CH}_{3}\right)_{2}\right), 1.55-1.77\left(\mathrm{~m}, 48 \mathrm{H}, \mathrm{CH}\left(\mathrm{CH}_{3}\right)_{2}\right), 2.90-2.95\left(\mathrm{~m}, 48 \mathrm{H}, \mathrm{CH}_{2} \mathrm{CH}_{2} \mathrm{~N}\right)$, $3.26\left(\mathrm{~d}, 54 \mathrm{H}, J=13.0, \mathrm{P}_{1} \mathrm{NCH}_{3}\right.$ and $\left.\mathrm{P}_{2} \mathrm{NCH}_{3}\right), 3.67-3.81\left(\mathrm{~m}, 48 \mathrm{H}, \mathrm{CHCH}_{2}\right), 3.90-$ $4.05\left(\mathrm{~m}, 96 \mathrm{H}, \mathrm{NCH}_{2}\right.$ and $\left.\mathrm{CH}_{2} \mathrm{CH}\right), 4.24\left(\mathrm{dd}, 48 \mathrm{H}, \mathrm{J}=\right.$ 8.3, 9.0, $\left.\mathrm{CH}_{2} \mathrm{CH}\right), 7.02-7.17$ (m, $\left.168 \mathrm{H}, \mathrm{CH}_{\text {aromatic }}\right), 7.57-7.62\left(\mathrm{~m}, 24 \mathrm{H}, \mathrm{CH}_{\text {aromatic }}\right), 7.58(\mathrm{~s}, 18 \mathrm{H}, \mathrm{CHN}) .{ }^{13} \mathrm{C}-\mathrm{NMR}$ (75.5 MHz, $\left.\mathrm{CDCl}_{3}\right): \delta=18.3\left(\mathrm{~s}, \mathrm{CH}\left(\mathrm{CH}_{3}\right)_{2}\right), 19.2\left(\mathrm{~s}, \mathrm{CH}\left(\mathrm{CH}_{3}\right)_{2}\right), 33.3\left(\mathrm{~s}, \mathrm{CH}\left(\mathrm{CH}_{3}\right)_{2}\right.$ and $\mathrm{N}_{1} \mathrm{CH}_{3}$ and $\mathrm{N}_{2} \mathrm{CH}_{3}$ ), 34.1 (s, $\mathrm{CH}_{2} \mathrm{CH}_{2} \mathrm{~N}$ ), 51.5 (s, $\mathrm{CH}_{2} \mathrm{CH}_{2} \mathrm{~N}$ ), $70.2\left(\mathrm{~s}, \mathrm{CHCH}_{2}\right.$ ), 71.6 (s, $\mathrm{CHCH}_{2}$ ), 121.6 (s, $C_{2}$ (ortho)), 122.1 (s, $C_{0}$ and $C_{1}$ (ortho)), 128.7 (s, $C_{0}$ and $C_{1}\left(\right.$ meta)), $130.6\left(\mathrm{~s}, C_{2}\right.$ (meta)), $132.9\left(\mathrm{~s}, C_{0}\right.$ and $C_{1}$ (para)), 136.5 (s, $C_{2}$ (para)), 138.8 
$\left(\mathrm{d},{ }^{3} J_{\mathrm{CP}}=14.0, C_{0} \mathrm{HN}\right.$ and $\left.C_{1} \mathrm{HN}\right), 149.4\left(\mathrm{~d},{ }^{2} J_{\mathrm{CP}}=7.0, C_{2}\right.$ (ipso)), $151.7\left(C_{0}\right.$ and $C_{1}$ (ipso)), $157.2(\mathrm{OCN})$.

Tessaraconta-oktakis-(4-\{2-[bis-(4-isopropyl-4,5-dihydro-oxazol-2-yl)-amino]ethyl\}-phenoxy)-cyclotriphosphazene $\left(G_{3} \cdot 4\right)$ :

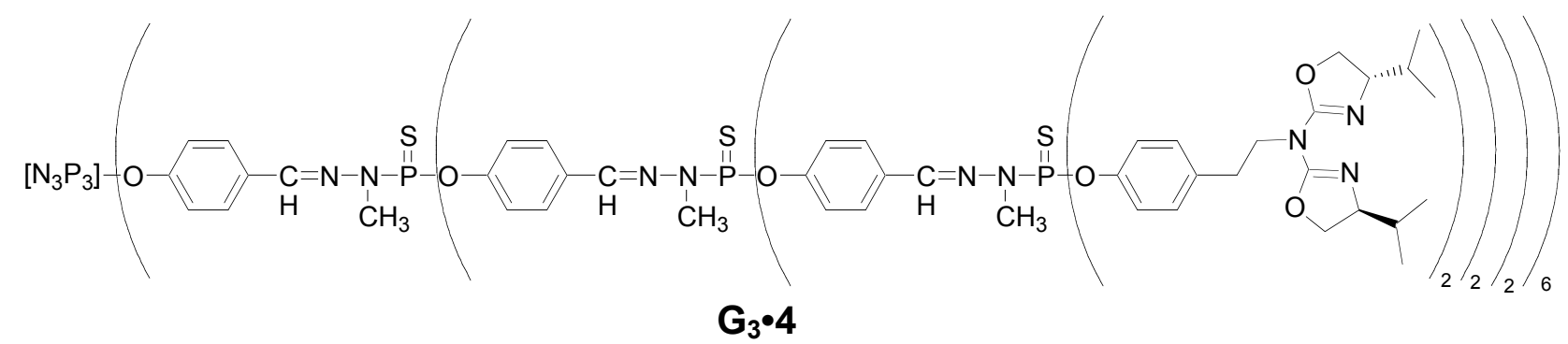

According to the general procedure, linker-bound aza-bis(oxazoline) 4 (0.05 g, 0.14 $\mathrm{mmol}), \mathrm{Cs}_{2} \mathrm{CO}_{3}(0.09 \mathrm{~g}, 0.28 \mathrm{mmol})$ and the dendrimer $\mathbf{G}_{3}(0.026 \mathrm{~g}, 0.0024 \mathrm{mmol})$ were reacted to form the product $\mathbf{G}_{3} \cdot 4$ as a white solid in $75 \%$ yield $(0.047 \mathrm{~g}, 0.0018$ $\mathrm{mmol})$.

${ }^{31} \mathrm{P}-\mathrm{NMR}\{1 \mathrm{H}\}\left(81 \mathrm{MHz}, \mathrm{CDCl}_{3}\right): \delta=11.7\left(\mathrm{~s}, \mathrm{P}_{0}\right), 66.1\left(\mathrm{~s}, \mathrm{P}_{1}\right.$ and $\left.\mathrm{P}_{2}\right), 66.5\left(\mathrm{~s}, \mathrm{P}_{3}\right)$. ${ }^{1} \mathrm{H}-\mathrm{NMR}\left(200 \mathrm{MHz}, \mathrm{CDCl}_{3}\right): \delta=0.85\left(\mathrm{~d}, 288 \mathrm{H}, J=6.2, \mathrm{CH}\left(\mathrm{CH}_{3}\right)_{2}\right), 0.90(\mathrm{~d}, 288 \mathrm{H}, J$ = 6.2, $\left.\mathrm{CH}\left(\mathrm{CH}_{3}\right)_{2}\right), 1.60-1.75\left(\mathrm{~m}, 96 \mathrm{H}, \mathrm{CH}\left(\mathrm{CH}_{3}\right)_{2}\right), 2.93-2.98\left(\mathrm{~m}, 96 \mathrm{H}, \mathrm{CH}_{2} \mathrm{CH}_{2} \mathrm{~N}\right)$, $3.32\left(\mathrm{~d}, 126 \mathrm{H}, J=13.0, \mathrm{P}_{1} \mathrm{NCH}_{3}\right.$ and $\mathrm{P}_{2} \mathrm{NCH}_{3}$ and $\left.\mathrm{P}_{3} \mathrm{NCH}_{3}\right)$, 3.74-3.88 $(\mathrm{m}, 96 \mathrm{H}$, $\left.\mathrm{CHCH}_{2}\right), 4.01-4.07\left(\mathrm{~m}, 192 \mathrm{H}, \mathrm{NCH}_{2}\right.$ and $\left.\mathrm{CH}_{2} \mathrm{CH}\right), 4.29(\mathrm{dd}, 96 \mathrm{H}, J=8.3,9.0$, $\mathrm{CH}_{2} \mathrm{CH}$ ), 7.11-7.18 (m, $336 \mathrm{H}, \mathrm{CH}_{\text {aromatic }}$ ), 7.62-7.70 (m, $\left.48 \mathrm{H}, \mathrm{CH}_{\text {aromatic }}\right), 7.60$ (s, 42 $\mathrm{H}, \mathrm{CHN}) .{ }^{13} \mathrm{C}-\mathrm{NMR}\left(75.5 \mathrm{MHz}, \mathrm{CDCl}_{3}\right): \delta=18.2\left(\mathrm{~s}, \mathrm{CH}\left(\mathrm{CH}_{3}\right)_{2}\right), 19.2\left(\mathrm{~s}, \mathrm{CH}\left(\mathrm{CH}_{3}\right)_{2}\right)$, 33.3 (s, $\mathrm{CH}\left(\mathrm{CH}_{3}\right)_{2}$ and $\mathrm{N}_{1} \mathrm{CH}_{3}$ and $\mathrm{N}_{2} \mathrm{CH}_{3}$ and $\left.\mathrm{N}_{3} \mathrm{CH}_{3}\right), 34.1\left(\mathrm{~s}, \mathrm{CH}_{2} \mathrm{CH}_{2} \mathrm{~N}\right), 51.5$ (s, $\mathrm{CH}_{2} \mathrm{CH}_{2} \mathrm{~N}$ ), 70.2 (s, $\mathrm{CHCH}_{2}$ ), 71.6 (s, $\mathrm{CHCH}_{2}$ ), 121.6 (s, $\mathrm{C}_{3}$ (ortho)), 122.2 (s, $\mathrm{C}_{0}$ and $C_{1}$ and $C_{2}$ (ortho)), 128.7 (s, $C_{0}$ and $C_{1}$ and $C_{2}$ (meta)), 130.6 (s, $C_{3}$ (meta)), 132.8 (s, $C_{0}$ and $C_{1}$ and $C_{2}$ (para)), 136.5 (s, $C_{3}$ (para)), $138.8\left(\mathrm{~d},{ }^{3} J_{\mathrm{CP}}=14.0, C_{0} \mathrm{HN}\right.$ and $C_{1} \mathrm{HN}$ and $C_{2} \mathrm{HN}$ ), $149.4\left(\mathrm{~d},{ }^{2} J_{\mathrm{CP}}=7.0, C_{3}(\right.$ ipso) $), 151.7\left(C_{0}\right.$ and $C_{1}$ and $C_{2}$ (ipso)), 157.2 $(\mathrm{OCN})$. 


\section{G1-Azide $\left(\mathrm{Ga}_{1}\right)$ :}

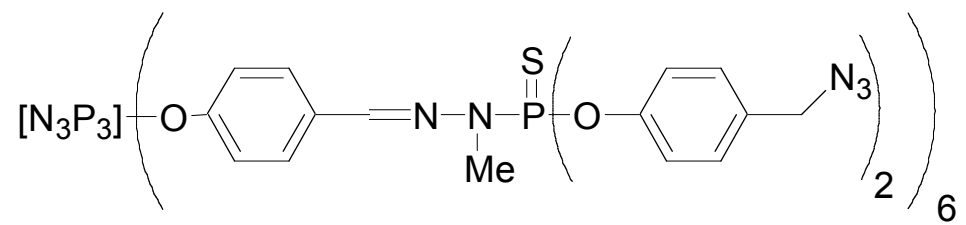

G1-chloride (200 mg, $0.065 \mathrm{mmol}$ ) was dissolved in DMF (20 mL) and sodium azide (252 mg, $3.88 \mathrm{mmol}$ ) was added. The mixture was stirred at room temperature for 40 $\mathrm{h}$, then it was poured onto water $(50 \mathrm{~mL})$ and extracted with $\mathrm{CH}_{2} \mathrm{Cl}_{2}(3 \times 20 \mathrm{~mL})$. The combined organic phases were dried over $\mathrm{MgSO}_{4}$, and the solvent was reduced to 2 $\mathrm{mL}$ via evaporation. After addition of pentane $(100 \mathrm{~mL})$, the product precipitated as a white solid (171 mg, $83 \%)$.

${ }^{31} \mathrm{P}-\mathrm{NMR}\left\{{ }^{1} \mathrm{H}\right\}\left(81 \mathrm{MHz}, \mathrm{CD}_{3} \mathrm{OD}\right): \delta=11.81\left(\mathrm{~s}, \mathrm{P}_{0}\right), 65.91\left(\mathrm{~s}, \mathrm{P}_{1}\right)$.

${ }^{1} \mathrm{H}-\mathrm{NMR}\left(300 \mathrm{MHz}, \mathrm{CD}_{3} \mathrm{OD}\right): \delta=7.63-7.57\left(\mathrm{~m}, 18 \mathrm{H}, \mathrm{C}_{0}{ }^{3}-\mathrm{H}, \mathrm{CHN}\right), 7.24-7.14(\mathrm{~m}, 48$ $\left.\mathrm{H}, \mathrm{C}_{1}{ }^{3}-\mathrm{H}, \mathrm{C}_{1}{ }^{2-} \mathrm{H}\right), 7.00\left(\mathrm{~d}, 12 \mathrm{H}, \mathrm{J}=8.51 \mathrm{~Hz}, \mathrm{C}_{0}{ }^{2}-H\right), 4.25\left(\mathrm{~s}, 24 \mathrm{H}, \mathrm{CH}_{2} \mathrm{~N}_{3}\right), 3.24$ (d, $\left.18 \mathrm{H}, J=10.2, \mathrm{P}_{1} \mathrm{NCH}_{3}\right) .{ }^{13} \mathrm{C}-\mathrm{NMR}\left(75.5 \mathrm{MHz}, \mathrm{CD}_{3} \mathrm{OD}\right): \delta=151.3\left(C_{0}{ }^{1}\right), 150.43$ $\left(C_{1}{ }^{1}\right), 138.7(\mathrm{CHN}), 132.8\left(C_{1}{ }^{4}\right), 132.2\left(C_{0}{ }^{4}\right), 129.5\left(C_{1}{ }^{3}\right), 128.3\left(C_{0}{ }^{3}\right), 121.8\left(C_{1}{ }^{2}\right)$, $121.5\left(\mathrm{C}_{0}^{2}\right), 54.1\left(\mathrm{CH}_{2} \mathrm{~N}_{3}\right), 33.0\left(\mathrm{CH}_{3}\right)$. IR: 3421, 2924, 2098, 1673, 1604, 1506 , $1193,1017,944 \mathrm{~cm}^{-1}$.

\section{G1-TriazolAzabox $\left(\mathrm{Ga}_{1} \cdot 5\right)$ :}

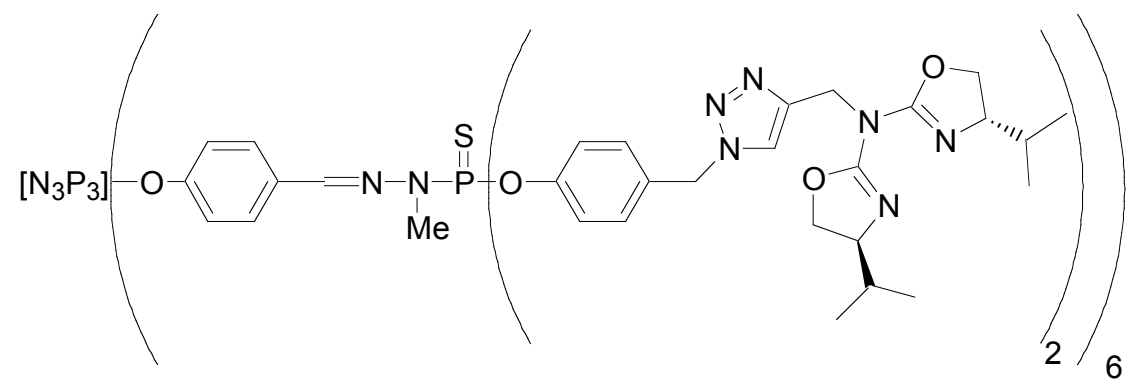

Under argon, G1-azide $\mathrm{Ga}_{1}(200 \mathrm{mg}, 0.063 \mathrm{mmol}$ ) and propargylazabisoxazoline 5 (230 mg, $0.830 \mathrm{mmol})$ were dissolved in dry THF $(10 \mathrm{~mL})$ and dry triethylamine $(2$ $\mathrm{mL})$. Then Cul $(0.083 \mathrm{mmol}, 16 \mathrm{mg})$ was added, and the mixture was stirred at room temperature for $20 \mathrm{~h}$. Afterwards, the solvent was evaporated, and the remaining solvent was dissolved in $\mathrm{CH}_{2} \mathrm{Cl}_{2}$. The organic phase was extracted with Titriplex IIIsolution (50 mg in $5 \mathrm{~mL}$ water) to remove the copper salts. Then the organic phase was dried over $\mathrm{MgSO}_{4}$ and reduced to $2 \mathrm{~mL}$. After addition of pentane, the product precipitated as a yellow powder $(328 \mathrm{mg}, 80 \%)$ 
${ }^{31} \mathrm{P}-\mathrm{NMR}\left\{{ }^{1} \mathrm{H}\right\}\left(81 \mathrm{MHz}, \mathrm{CDCl}_{3}\right): \delta=65.94\left(\mathrm{~s}, \mathrm{P}_{1}\right), 11.66\left(\mathrm{~s}, \mathrm{P}_{0}\right)$.

${ }^{1} \mathrm{H}-\mathrm{NMR}\left(200 \mathrm{MHz}, \mathrm{CDCl}_{3}\right): \delta=7.70-7.50\left(\mathrm{~m}, 30 \mathrm{H}, \mathrm{CH}_{\text {triazol }}, \mathrm{CHN}, \mathrm{C}_{0}{ }^{3} \mathrm{H}\right), 7.20-6.90$ ( $\mathrm{m}, 60 \mathrm{H}, \mathrm{CH}_{\mathrm{ar}}$ ), 5.38 (s, $24 \mathrm{H}, \mathrm{CH}_{2}$ ), 5.11 and 5.05 (AB syst., $24 \mathrm{H}, J=15.5 \mathrm{~Hz}$, $\mathrm{CH}_{2} \mathrm{~N}$ ), $4.33\left(\mathrm{dd}, 24 \mathrm{H}, \mathrm{J}=8.7,8.7 \mathrm{~Hz}, \mathrm{CH}_{2} \mathrm{CH}\right.$ ), $4.06(\mathrm{dd}, 24 \mathrm{H}, J=6.9,8.7 \mathrm{~Hz}$, $\mathrm{CH}_{2} \mathrm{CH}$ ), $3.83\left(\mathrm{~m}, 24 \mathrm{H}, \mathrm{CH}_{2} \mathrm{CH}\right), 3.24(\mathrm{br} \mathrm{d}, 18 \mathrm{H}, \mathrm{J}=10.6 \mathrm{~Hz}, \mathrm{NMe}), 1.95-1.50(\mathrm{~m}$, $24 \mathrm{H}, \mathrm{CH} / \mathrm{Pr}$ ), 0.81 (d, $\left.72 \mathrm{H}, \mathrm{J}=6.70 \mathrm{~Hz}, \mathrm{CH}_{3}\right), 0.74\left(\mathrm{~d}, 72 \mathrm{H}, \mathrm{J}=6.60 \mathrm{~Hz}, \mathrm{CH}_{3}\right.$ ).

${ }^{13} \mathrm{C}$-NMR $\left(50 \mathrm{MHz}, \mathrm{CDCl}_{3}\right): \delta=156.8(\mathrm{NCNO}), 151.3\left(\mathrm{C}_{0}{ }^{1}\right), 150.43\left(C_{1}{ }^{1}\right), 138.7$ $(\mathrm{CHN}), 132.8\left(C_{0}{ }^{4}\right), 132.2\left(C_{1}{ }^{4}\right), 129.5\left(C_{1}{ }^{3}\right), 129.4\left(C_{\text {Triazol }}\right), 128.3\left(C_{0}{ }^{3}\right), 121.8$ $\left(\mathrm{C}_{1}^{2}\right), 121.5\left(\mathrm{C}_{0}^{2}\right), 71.5\left(\mathrm{CH}_{2} \mathrm{~N}\right), 70.6\left(\mathrm{CH}_{2} \mathrm{O}\right), 69.8\left(\mathrm{CH}_{2} \mathrm{CH}\right), 45.1\left(\mathrm{CH}_{2} \mathrm{~N}\right), 33.0$

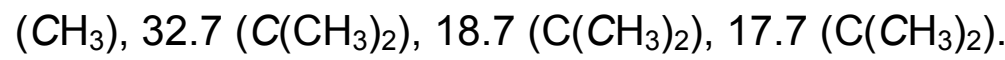

IR: $3432,2959,1640,150,1428,1196,1162,942,787 \mathrm{~cm}^{-1}$.

\section{G2-Alcohol:}

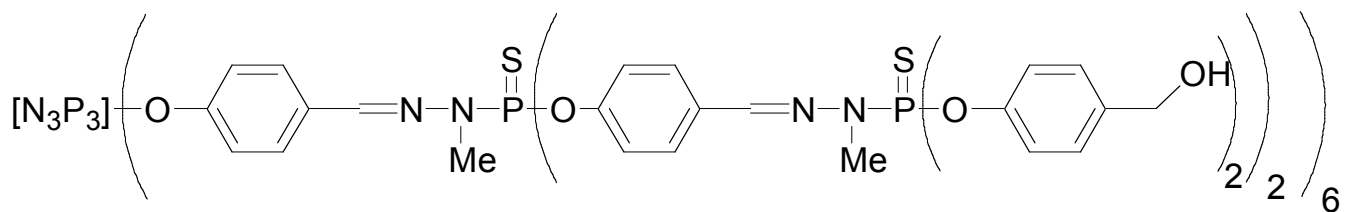

Under argon, dendrimer $\mathbf{G f}_{2}(1.00 \mathrm{~g}, 0.146 \mathrm{mmol})$ was dissolved in dry $\mathrm{CH}_{2} \mathrm{Cl}_{2}(40$ $\mathrm{mL})$ and cooled to $0^{\circ} \mathrm{C}$. Then $\mathrm{BH}_{3}-\mathrm{SMe}_{2}\left(1.75 \mathrm{~mL}\right.$ of a $1 \mathrm{M}$ solution in $\mathrm{CH}_{2} \mathrm{Cl}_{2}, 1.75$ $\mathrm{mmol}$ ) was added dropwise, and the mixture was stirred another $20 \mathrm{~min}$ at $0^{\circ} \mathrm{C}$ and then overnight at room temperature. The reaction was quenched via dropwise addition to methanol $(100 \mathrm{~mL})$ and the solvent was evaporated. Then the residue was again dissolved in methanol and the solvent was evaporated. The remaining solid was washed with pentane to give the title compound in $970 \mathrm{mg}, 96.3 \%$.

\section{G2-Chloride:}

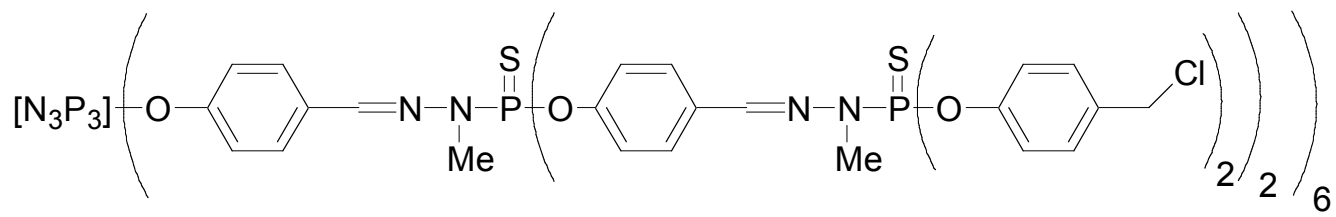

Under argon, dendrimer G2-alcohol $(0.141 \mathrm{mmol}, 970 \mathrm{mg})$ was dissolved in dry $\mathrm{CH}_{2} \mathrm{Cl}_{2}(40 \mathrm{~mL})$ and cooled to $0^{\circ} \mathrm{C}$. Then thionylchloride $(3.716 \mathrm{mmol}, 271 \mu \mathrm{L})$ was added drowise, and the solution was slowly warmed to room temperature overnight 
while stirring. The solvent was evaporated, and the remaining solid was dissolved in $\mathrm{CH}_{2} \mathrm{Cl}_{2}(10 \mathrm{~mL})$ and again evaporated (2 times). The product was obtained by dilution in $\mathrm{CH}_{2} \mathrm{Cl}_{2}(2 \mathrm{~mL})$ and precipitation via addition of pentane as a white solid in $800 \mathrm{mg}, 77.5 \%$ yield.

${ }^{31} \mathrm{P}-\mathrm{NMR}\left\{{ }^{1} \mathrm{H}\right\}\left(101.25 \mathrm{MHz}, \mathrm{CDCl}_{3}\right): \delta=11.96\left(\mathrm{~s}, \mathrm{P}_{0}\right), 65.65\left(\mathrm{~s}, \mathrm{P}_{1}\right), 65.83\left(\mathrm{~s}, \mathrm{P}_{2}\right)$.

${ }^{1} \mathrm{H}-\mathrm{NMR}\left(250 \mathrm{MHz}, \mathrm{CDCl}_{3}\right): \delta=7.63-7.58\left(\mathrm{~m}, 54 \mathrm{H}, \mathrm{CHN}, \mathrm{C}_{\mathrm{ar}} H\right), 7.31-7.17(\mathrm{~m}, 120$ $\left.\mathrm{H}, \mathrm{C}_{\mathrm{ar}} \mathrm{H}\right), 7.01-6.85\left(\mathrm{~m}, 12 \mathrm{H}, \mathrm{C}_{0} \mathrm{H}\right), 4.46\left(\mathrm{~s}, 48 \mathrm{H}, \mathrm{CH}_{2} \mathrm{Cl}\right), 3.35-3.17\left(\mathrm{~m}, 54 \mathrm{H}, \mathrm{NCH}_{3}\right)$. ${ }^{13} \mathrm{C}-\mathrm{NMR}\left(75.5 \mathrm{MHz}, \mathrm{CDCl}_{3}\right): \delta=151.3\left(C_{0}{ }^{1}\right), 150.4\left(C_{1}{ }^{1}\right), 150.3\left(C_{2}{ }^{1}\right), 138.9(\mathrm{CHN})$, $138.6(\mathrm{CHN}), 132.8\left(C_{2}{ }^{4}\right), 132.2\left(C_{1}{ }^{4}\right), 132.1\left(C_{0}{ }^{4}\right), 129.9\left(C_{2}{ }^{3}\right), 128.3\left(C_{1}{ }^{3}, C_{0}{ }^{3}\right), 121.7$ $\left(C_{2}{ }^{2}\right), 121.6\left(C_{1}{ }^{2}, C_{0}{ }^{2}\right), 45.5\left(C_{2} \mathrm{~N}_{3}\right), 33.1\left(C_{3}\right), 32.9\left(C_{3}\right)$. IR: 3450, 1604, 1505, $1467,1193,1162,1017,924,838,783 \mathrm{~cm}^{-1}$.

\section{G2-Azide $\left(\mathrm{Ga}_{2}\right)$ :}

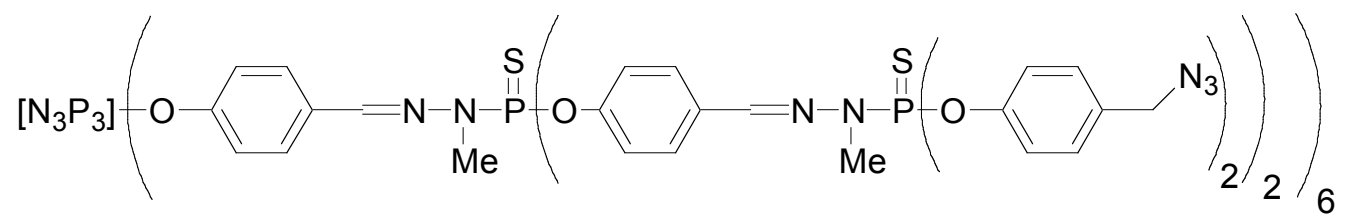

G2-chloride (800 mg, $0.109 \mathrm{mmol}$ ) was dissolved in DMF (20 mL) and sodium azide (850 mg, $6.54 \mathrm{mmol}$ ) was added. The mixture was stirred at room temperature for 40 $\mathrm{h}$, then it was poured onto water $(50 \mathrm{~mL})$ and extracted with $\mathrm{CH}_{2} \mathrm{Cl}_{2}(3 \times 20 \mathrm{~mL})$. The combined organic phases were dried over $\mathrm{MgSO}_{4}$, and the solvent was reduced to 2 $\mathrm{mL}$ via evaporation. After addition of pentane/diethylether $1: 1(100 \mathrm{~mL})$, the product precipitated as a white solid (680 $\mathrm{mg}, 83.2 \%)$.

${ }^{31}$ P-NMR (81 MHz, CDCl 3 ): $\delta=11.92\left(\mathrm{~s}, \mathrm{P}_{0}\right), 65.90$ (s, $\left.\mathrm{P}_{1}\right), 65.90$ (s, $\left.\mathrm{P}_{2}\right) \mathrm{ppm}$.

${ }^{1} \mathrm{H}-\mathrm{NMR}\left(200 \mathrm{MHz}_{\mathrm{C}} \mathrm{CDCl}_{3}\right): \delta=7.63-7.50\left(\mathrm{~m}, 54 \mathrm{H}, \mathrm{CHN}, \mathrm{C}_{\mathrm{ar}} \mathrm{H}\right), 7.25-7.10(\mathrm{~m}, 120$ $\left.\mathrm{H}, \mathrm{C}_{\mathrm{ar}} \mathrm{H}\right), 6.95-6.80\left(\mathrm{~m}, 12 \mathrm{H}, \mathrm{C}_{0}{ }^{2} \mathrm{H}\right), 4.22\left(\mathrm{~s}, 48 \mathrm{H}, \mathrm{CH}_{2} \mathrm{~N}_{3}\right), 3.30-3.10(\mathrm{~m}, 54 \mathrm{H}$, $\left.\mathrm{NCH}_{3}\right) \cdot{ }^{13} \mathrm{C}-\mathrm{NMR}\left(50 \mathrm{MHz}, \mathrm{CDCl}_{3}\right): \delta=151.3\left(\mathrm{C}_{0}{ }^{1}\right), 150.4\left(C_{1}{ }^{1}\right), 150.3\left(C_{2}{ }^{1}\right), 138.8$ $(\mathrm{CHN}), 138.7(\mathrm{CHN}), 132.8\left(C_{2}{ }^{4}\right), 132.2\left(C_{1}{ }^{4}\right), 132.1\left(C_{0}{ }^{4}\right), 129.4\left(C_{2}{ }^{3}\right), 128.3$ $\left(C_{1}{ }^{3}, C_{0}{ }^{3}\right), 121.8\left(C_{2}{ }^{2}\right), 121.5\left(C_{1}{ }^{2}, C_{0}{ }^{2}\right), 54.1\left(C_{2} \mathrm{~N}_{3}\right), 33.1\left(C_{3}\right), 33.0\left(C H_{3}\right)$. IR: $3444,2097,1604,1505,1467,1191,1160,1017,921,835,782 \mathrm{~cm}^{-1}$. 


\section{G2-TriazolAzabox $\left(\mathrm{Ga}_{2} \cdot 5\right)$ :}

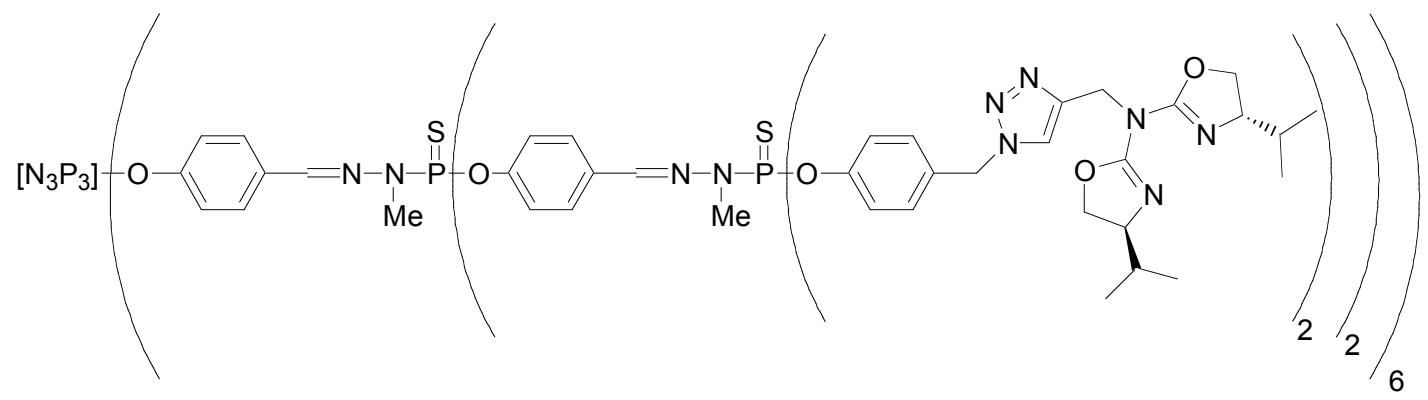

Under argon, G2-azide $\mathbf{G a}_{2}(830 \mathrm{mg}, 0.110 \mathrm{mmol}$ ) and propargylazabisoxazoline 5 (955 mg, $3.44 \mathrm{mmol}$ ) were dissolved in dry THF $(10 \mathrm{~mL})$ and dry triethylamine $(2 \mathrm{~mL})$. Then Cul $(0.083 \mathrm{mmol}, 16 \mathrm{mg})$ was added, and the mixture was stirred at room temperature for $20 \mathrm{~h}$. Afterwards, the solvent was evaporated, and the remaining solvent was dissolved in $\mathrm{CH}_{2} \mathrm{Cl}_{2}$. The organic phase was extracted with Titriplex IIIsolution (50 mg in $5 \mathrm{~mL}$ water) to remove the copper salts. Then the organic phase was dried over $\mathrm{MgSO}_{4}$ and reduced to $2 \mathrm{~mL}$. After addition of pentane, the product precipitated as a yellow powder (1250 $\mathrm{mg}, 88 \%)$

${ }^{31} \mathrm{P}-\mathrm{NMR}\left(81 \mathrm{MHz}, \mathrm{CDCl}_{3}\right): \delta=11.7\left(\mathrm{~s}, \mathrm{P}_{0}\right), 65.8\left(\mathrm{~s}, \mathrm{P}_{1}\right), 65.9\left(\mathrm{~s}, \mathrm{P}_{2}\right) \mathrm{ppm}$.

${ }^{1} \mathrm{H}-\mathrm{NMR}\left(200 \mathrm{MHz}, \mathrm{CDCl}_{3}\right): \delta=7.85-7.50\left(\mathrm{~m}, 78 \mathrm{H}, \mathrm{CH}_{\text {triazol }}, \mathrm{CH}_{\mathrm{ar}}, \mathrm{CHN}\right), 7.25-7.00$ (m, $132 \mathrm{H}, \mathrm{CH}_{\mathrm{ar}}$ ), 5.37 (s, $48 \mathrm{H}, \mathrm{NCH}_{2} \mathrm{C}_{\mathrm{ar}}$ ), 5.09 and 5.05 (AB syst., $48 \mathrm{H}, J=15.7$ $\mathrm{Hz}, \mathrm{N}_{\text {azabo }} \mathrm{CH}_{2}$ ), 4.31 (dd, $48 \mathrm{H}, J=8.7,8.7 \mathrm{~Hz}, \mathrm{CH}_{2} \mathrm{CH}$ ), 4.05 (dd, $48 \mathrm{H}, J=6.9,8.7$ $\left.\mathrm{Hz}, \mathrm{CH}_{2} \mathrm{CH}\right), 3.77\left(\mathrm{~m}, 48 \mathrm{H}, \mathrm{CH}_{2} \mathrm{CH}\right), 3.30-3.20\left(\mathrm{~m}, 54 \mathrm{H}, \mathrm{NCH}_{3}\right), 1.70-1.54(\mathrm{~m}, 48 \mathrm{H}$, $\left.\mathrm{CH}\left(\mathrm{CH}_{3}\right)_{2}\right), 0.80\left(\mathrm{~d}, 144 \mathrm{H}, J=6.7 \mathrm{~Hz}, \mathrm{C}\left(\mathrm{CH}_{3}\right)_{2}\right), \quad 0.73(\mathrm{~d}, 144 \mathrm{H}, J=6.6 \mathrm{~Hz}$, $\left.\mathrm{C}\left(\mathrm{CH}_{3}\right)_{2}\right) \cdot{ }^{13} \mathrm{C}-\mathrm{NMR}\left(63 \mathrm{MHz}, \mathrm{CDCl}_{3}\right): \delta=156.8(\mathrm{NCNO}), 151.3\left(\mathrm{C}_{0}{ }^{1}\right), 150.4\left(\mathrm{C}_{1}{ }^{1}\right)$, $150.3\left(C_{2}{ }^{1}\right), 138.8(\mathrm{CHN}), 138.7(\mathrm{CHN}), 132.8\left(\mathrm{C}_{0}{ }^{4}, C_{1}{ }^{4}\right), 132.2\left(C_{2}{ }^{4}\right), 129.5\left(C_{2}{ }^{3}\right)$, $129.4\left(\mathrm{CH}_{\text {Triazol }}\right), 128.3\left(\mathrm{C}_{0}^{3}, \mathrm{C}_{1}{ }^{3}\right), 121.8\left(\mathrm{C}_{1}{ }^{2}\right), 121.5\left(\mathrm{C}_{0}^{2}, \mathrm{C}_{1}{ }^{2}\right), 71.5\left(\mathrm{CH}_{2} \mathrm{~N}\right), 70.6$

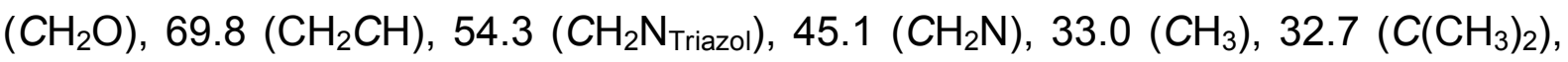

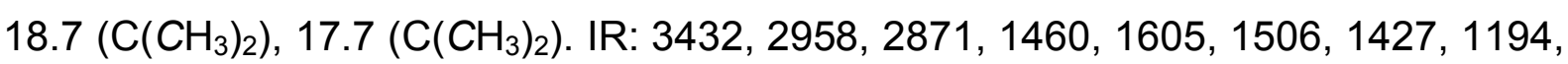
$1162,933,839,784 \mathrm{~cm}^{-1}$.

\section{G3-Alcohol (G3-OH):}

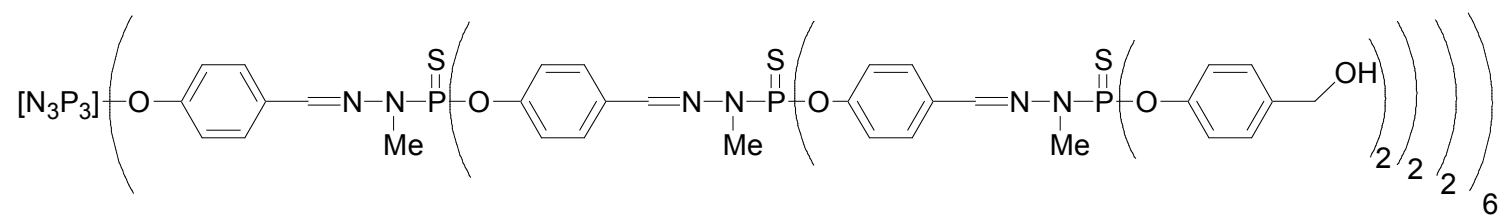

Under argon, dendrimer $\mathbf{G f}_{3}$ (350 $\mathrm{mg}, 0.0236 \mathrm{mmol}$ ) was dissolved in dry $\mathrm{CH}_{2} \mathrm{Cl}_{2}$ (40 $\mathrm{mL})$ and cooled to $0^{\circ} \mathrm{C}$. Then $\mathrm{BH}_{3}-\mathrm{SMe}_{2}\left(1.13 \mathrm{~mL}\right.$ of a $1 \mathrm{M}$ solution in $\mathrm{CH}_{2} \mathrm{Cl}_{2}, 1.13$ 
mmol) was added dropwise, and the mixture was stirred another 20 min at $0^{\circ} \mathrm{C}$ and then overnight at room temperature. The reaction was quenched via dropwise addition to methanol $(100 \mathrm{~mL})$ and the solvent was evaporated. Then the residue was again dissolved in methanol and the solvent was evaporated. The remaining solid was washed with pentane to give the title compound in $320 \mathrm{mg}, 90.8 \%$.

${ }^{1} \mathrm{H}-\mathrm{NMR}\left(250 \mathrm{MHz}, \mathrm{CDCl}_{3}\right): \delta=7.63-7.58\left(\mathrm{~m}, 126 \mathrm{H}, \mathrm{CHN}, \mathrm{C}_{\mathrm{ar}} \mathrm{H}\right), 7.31-7.17(\mathrm{~m}, 264$ $\left.\mathrm{H}, \mathrm{C}_{\mathrm{ar}} \mathrm{H}\right), 7.01-6.85\left(\mathrm{~m}, 12 \mathrm{H}, \mathrm{C}_{0} \mathrm{H}\right), 4.35\left(\mathrm{~s}, 96 \mathrm{H}, \mathrm{CH}_{2} \mathrm{Cl}\right), 3.35-3.17(\mathrm{~m}, 126 \mathrm{H}$, $\mathrm{NCH}_{3}$.

\section{G3-Chloride:}

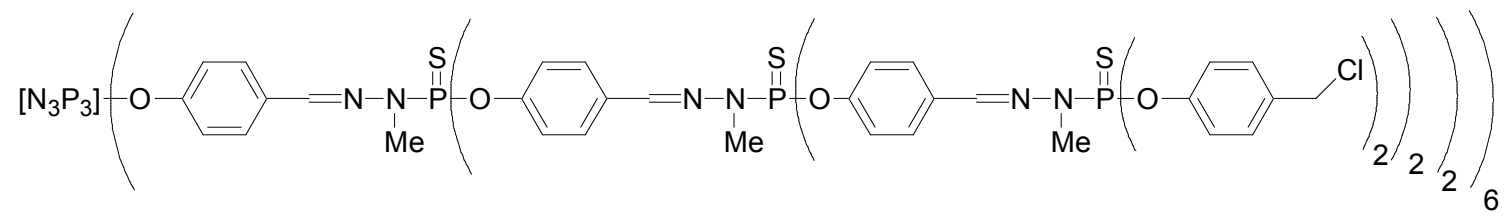

Under argon, dendrimer G3-OH $(0.0142 \mathrm{mmol}, 212 \mathrm{mg})$ was dissolved in dry $\mathrm{CH}_{2} \mathrm{Cl}_{2}$ $(40 \mathrm{~mL})$ and cooled to $0{ }^{\circ} \mathrm{C}$. Then thionylchloride $(0.750 \mathrm{mmol}, 55 \mu \mathrm{L})$ was added dropwise, and the solution was slowly warmed to room temperature overnight while stirring. The solvent was evaporated, and the remaining solid was dissolved in $\mathrm{CH}_{2} \mathrm{Cl}_{2}(10 \mathrm{~mL})$ and again evaporated (2 times). The product was obtained by dilution in $\mathrm{CH}_{2} \mathrm{Cl}_{2}(2 \mathrm{~mL})$ and precipitation via addition of pentane as a white solid in $208 \mathrm{mg}, 93 \%$ yield.

${ }^{31} \mathrm{P}-N M R\left\{{ }^{1} \mathrm{H}\right\}\left(101.25 \mathrm{MHz}, \mathrm{CDCl}_{3}\right): \delta=8.46\left(\mathrm{~s}, \mathrm{P}_{0}\right), 65.7\left(\mathrm{~s}, \mathrm{P}_{1}, \mathrm{P}_{2}, \mathrm{P}_{3}\right)$.

${ }^{1} \mathrm{H}-\mathrm{NMR}\left(250 \mathrm{MHz}_{\mathrm{CDCl}}\right): \delta=7.63-7.58\left(\mathrm{~m}, 126 \mathrm{H}, \mathrm{CHN}, \mathrm{C}_{\mathrm{ar}} \mathrm{H}\right), 7.31-6.85(\mathrm{~m}, 276$ $\left.\mathrm{H}, \mathrm{C}_{\mathrm{ar}} \mathrm{H}\right), 4.41\left(\mathrm{~s}, 96 \mathrm{H}, \mathrm{CH}_{2} \mathrm{Cl}\right), 3.35-3.17\left(\mathrm{~m}, 126 \mathrm{H}, \mathrm{NCH}_{3}\right) . \mathrm{IR}: 3450,1604,1505$, $1467,1193,1162,1017,924,838,783 \mathrm{~cm}^{-1}$.

\section{G3-Azide $\left(\mathrm{Ga}_{3}\right)$ :}

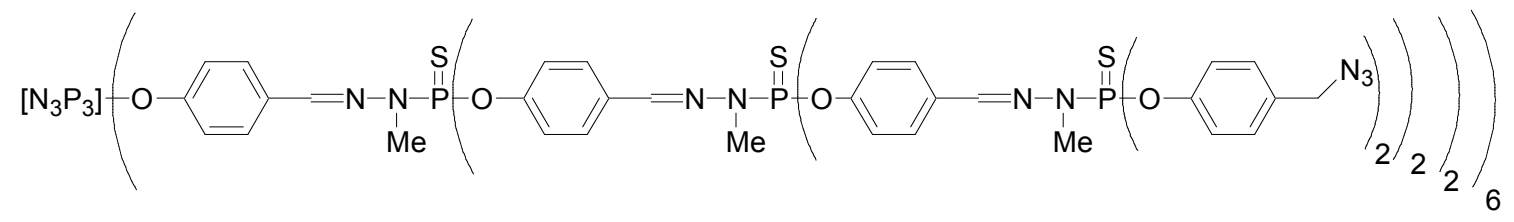


G3-chloride (200 mg, $0.0126 \mathrm{mmol}$ ) was dissolved in DMF (20 mL) and sodium azide (197 mg, $3.037 \mathrm{mmol}$ ) was added. The mixture was stirred at room temperature for $40 \mathrm{~h}$, then it was poured onto water $(50 \mathrm{~mL})$ and extracted with $\mathrm{CH}_{2} \mathrm{Cl}_{2}(3 \times 20 \mathrm{~mL})$. The combined organic phases were dried over $\mathrm{MgSO}_{4}$, and the solvent was reduced to $2 \mathrm{~mL}$ via evaporation. After addition of pentane/diethylether $1: 1(100 \mathrm{~mL})$, the product precipitated as a white solid ( $160 \mathrm{mg}, 77.1 \%$ ).

${ }^{31} \mathrm{P}-\mathrm{NMR}\left\{{ }^{1} \mathrm{H}\right\}\left(81 \mathrm{MHz}, \mathrm{CDCl}_{3}\right): \delta=11.70\left(\mathrm{~s}, \mathrm{P}_{0}\right), 65.77\left(\mathrm{~s}, \mathrm{P}_{1}\right), 65.77\left(\mathrm{~s}, \mathrm{P}_{2}\right), 65.77$ (s, $\left.\mathrm{P}_{3}\right) \mathrm{ppm}$.

${ }^{1} \mathrm{H}-\mathrm{NMR}\left(200 \mathrm{MHz}, \mathrm{CDCl}_{3}\right): \delta=7.70-7.50\left(\mathrm{~m}, 126 \mathrm{H}, \mathrm{CHN}, \mathrm{C}_{\mathrm{ar}} H\right), 7.20-6.90(\mathrm{~m}, 276$ $\left.\mathrm{H}, \mathrm{C}_{\mathrm{ar}} \mathrm{H}\right), 4.19\left(\mathrm{~s}, 96 \mathrm{H}, \mathrm{CH}_{2} \mathrm{~N}_{3}\right), 3.30-3.20(\mathrm{~m}, 126 \mathrm{H}, \mathrm{NCH}) .{ }^{13} \mathrm{C}-\mathrm{NMR}(75.5 \mathrm{MHz}$, $\left.\mathrm{CDCl}_{3}\right): \delta=151.3\left(C_{1}{ }^{1}\right), 150.5\left(C_{2}{ }^{1}\right), 150.4\left(C_{3}{ }^{1}\right), 139.0(\mathrm{CHN}), 132.6\left(C_{3}{ }^{4}\right), 132.2$ $\left(C_{2}{ }^{4}\right), 129.4\left(C_{3}{ }^{3}, C_{2}{ }^{3}\right), 128.3\left(C_{1}{ }^{3}, C_{0}{ }^{3}\right), 121.8\left(C_{3}{ }^{2}, C_{2}{ }^{2}\right), 121.7\left(C_{1}{ }^{2}, C_{0}{ }^{2}\right), 54.0$ $\left(\mathrm{CH}_{2} \mathrm{~N}_{3}\right), 33.1\left(\mathrm{CH}_{3}\right), 32.9\left(\mathrm{CH}_{3}\right)$. IR: 3445, 2934, 2098, 1603, 1506, 1467, 1292, $1192,1161,1016,922,837,782$

$\mathrm{cm}^{-1}$.

\section{G3-TriazolAzabox $\left(\mathrm{Ga}_{3} \cdot 5\right)$ :}

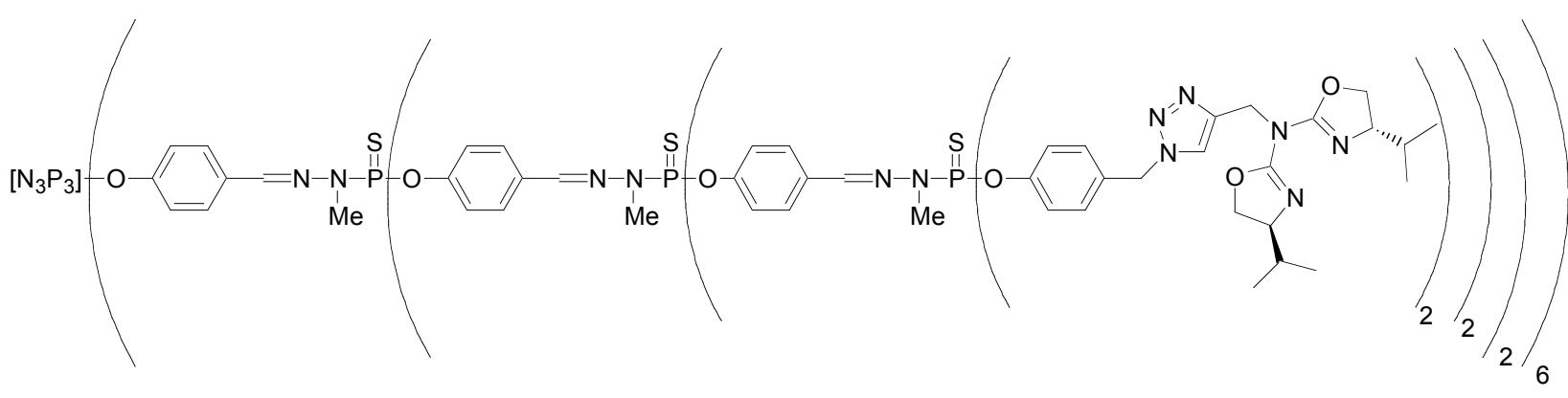

Under argon, G3-azide $\mathrm{Ga}_{3}\left(160 \mathrm{mg}, 9.7510^{-3} \mathrm{mmol}\right.$ ) and propargyl-azabisoxazoline 5 (200 mg, $0.721 \mathrm{mmol})$ were dissolved in dry THF $(10 \mathrm{~mL})$ and dry triethylamine (2 $\mathrm{mL})$. Then Cul $(0.083 \mathrm{mmol}, 16 \mathrm{mg})$ was added, and the mixture was stirred at room temperature for $20 \mathrm{~h}$. Afterwards, the solvent was evaporated, and the remaining solvent was dissolved in $\mathrm{CH}_{2} \mathrm{Cl}_{2}$. The organic phase was extracted with Titriplex IIIsolution (50 mg in $5 \mathrm{~mL}$ water) to remove the copper salts. Then the organic phase was dried over $\mathrm{MgSO}_{4}$ and reduced to $2 \mathrm{~mL}$. After addition of pentane, the product precipitated as a yellow powder $(243 \mathrm{mg}, 84 \%)$ 
${ }^{31} \mathrm{P}-\mathrm{NMR}\left\{{ }^{1} \mathrm{H}\right\}\left(81 \mathrm{MHz}, \mathrm{CDCl}_{3}\right): \delta=11.70\left(\mathrm{~s}, \mathrm{P}_{0}\right), 65.7\left(\mathrm{~s}, \mathrm{P}_{1}, \mathrm{P}_{2}, \mathrm{P}_{3}\right)$.

${ }^{1} \mathrm{H}-\mathrm{NMR}\left(200 \mathrm{MHz}, \mathrm{CDCl}_{3}\right): \delta=7.85-7.50\left(\mathrm{~m}, 174 \mathrm{H}, \mathrm{CH}_{\text {triazol }}, \mathrm{CHN}, \mathrm{CH}_{\mathrm{ar}}\right), 7.25-7.00$ (m, $276 \mathrm{H}, \mathrm{CH}_{\text {arr }}$ ), 5.37 (bs, $96 \mathrm{H}, \mathrm{NCH}_{2} \mathrm{C}_{\text {ar }}$ ), 5.06 (m, $96 \mathrm{H}, \mathrm{N}_{\text {azabox }} \mathrm{CH}_{2}$ ), 4.31 (dd, 96 $\left.\mathrm{H}, J=8.7,8.7 \mathrm{~Hz}, \mathrm{CH}_{2} \mathrm{CH}\right), 4.05\left(\mathrm{dd}, 96 \mathrm{H}, J=6.8,8.7 \mathrm{~Hz}, \mathrm{CH}_{2} \mathrm{CH}\right), 3.77(\mathrm{~m}, 96 \mathrm{H}$, $\left.\mathrm{CH}_{2} \mathrm{CH}\right), 3.30-3.20\left(\mathrm{~m}, 126 \mathrm{H}, \mathrm{NCH}_{3}\right), 1.70-1.54\left(\mathrm{~m}, 96 \mathrm{H}, \mathrm{CHC}\left(\mathrm{CH}_{3}\right)_{2}\right), 0.80(\mathrm{~d}, 288$ $\left.\mathrm{H}, J=6.7 \mathrm{~Hz}, \mathrm{C}\left(\mathrm{CH}_{3}\right)_{2}\right), 0.73\left(\mathrm{~d}, 288 \mathrm{H}, J=6.6 \mathrm{~Hz}, \mathrm{C}\left(\mathrm{CH}_{3}\right)_{2}\right)$. IR: 3443, 2959, 1642, $1506,1428,1384,1194,1162,921,783 \mathrm{~cm}^{-1}$. 
4-tert-Butyl-dimethyl-silyloxy-phenethyl iodide (2):
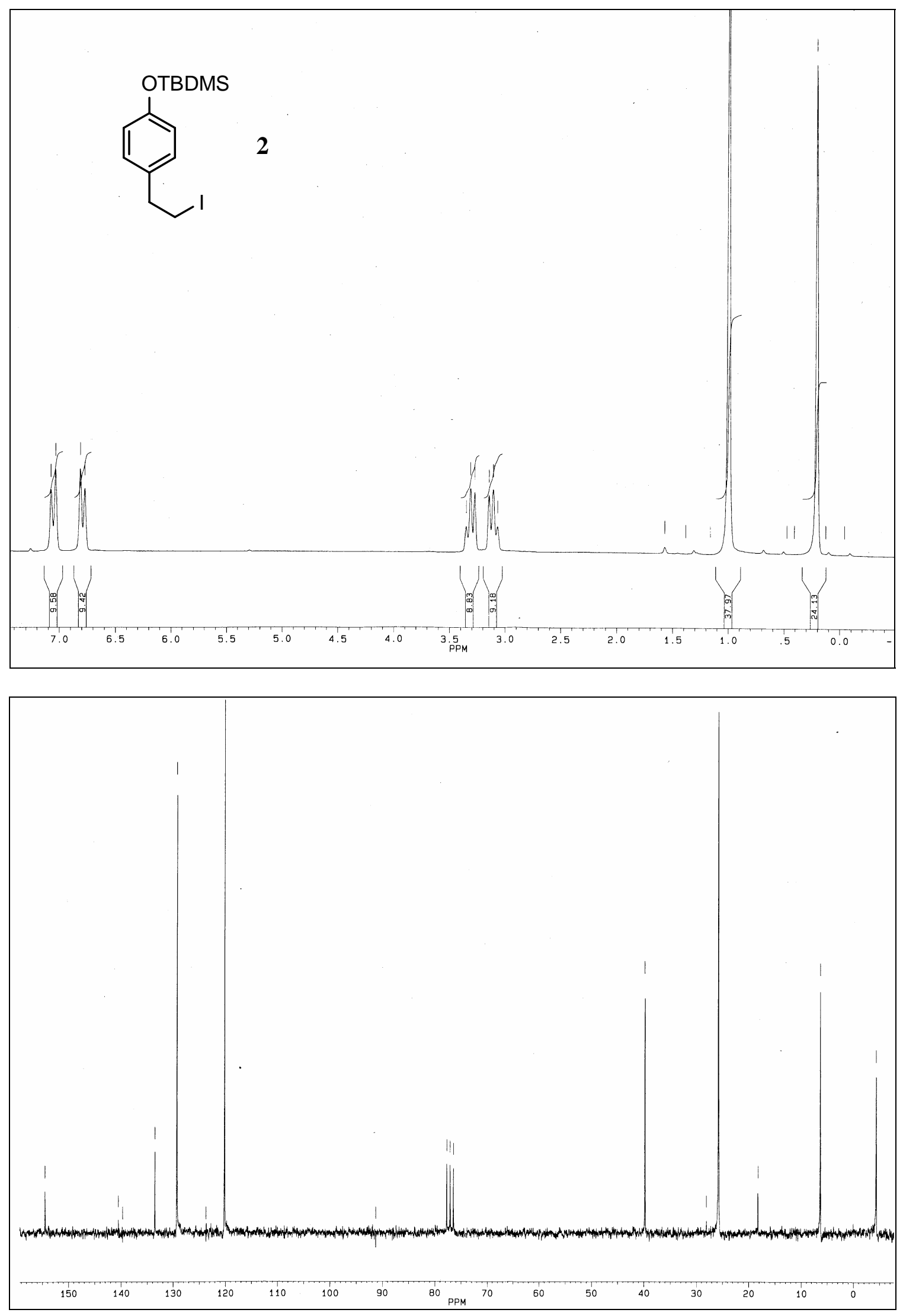
\{2-[4-(tert-Butyl-dimethyl-silanyloxy)-phenyl]-ethyl\}-bis-(4-isopropyl-4,5dihydro-oxazol-2-yl)-amine (3):
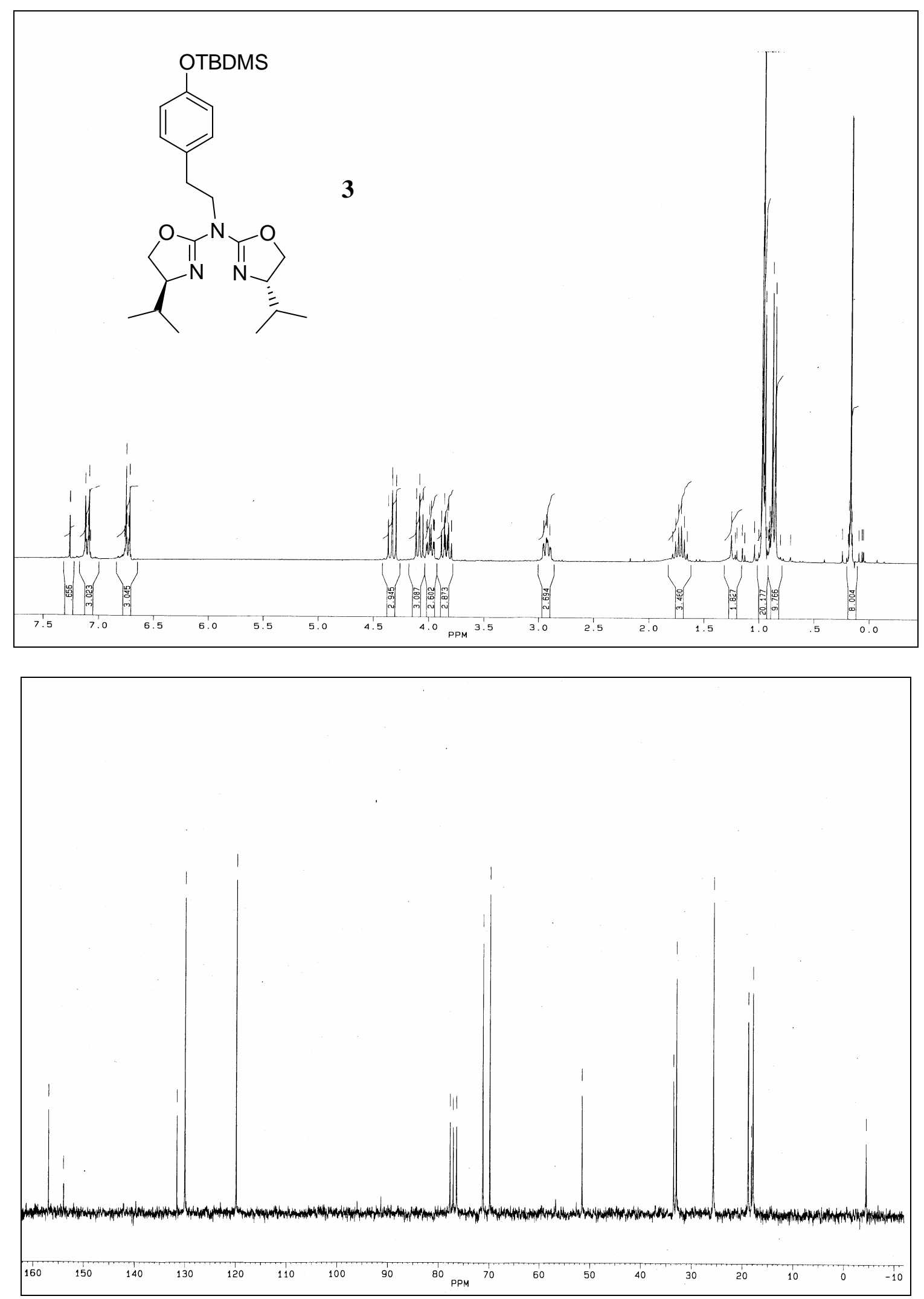
4-\{2-[Bis-(4-isopropyl-4,5-dihydro-oxazol-2-yl)-amino]-ethyl\}-phenol (4):
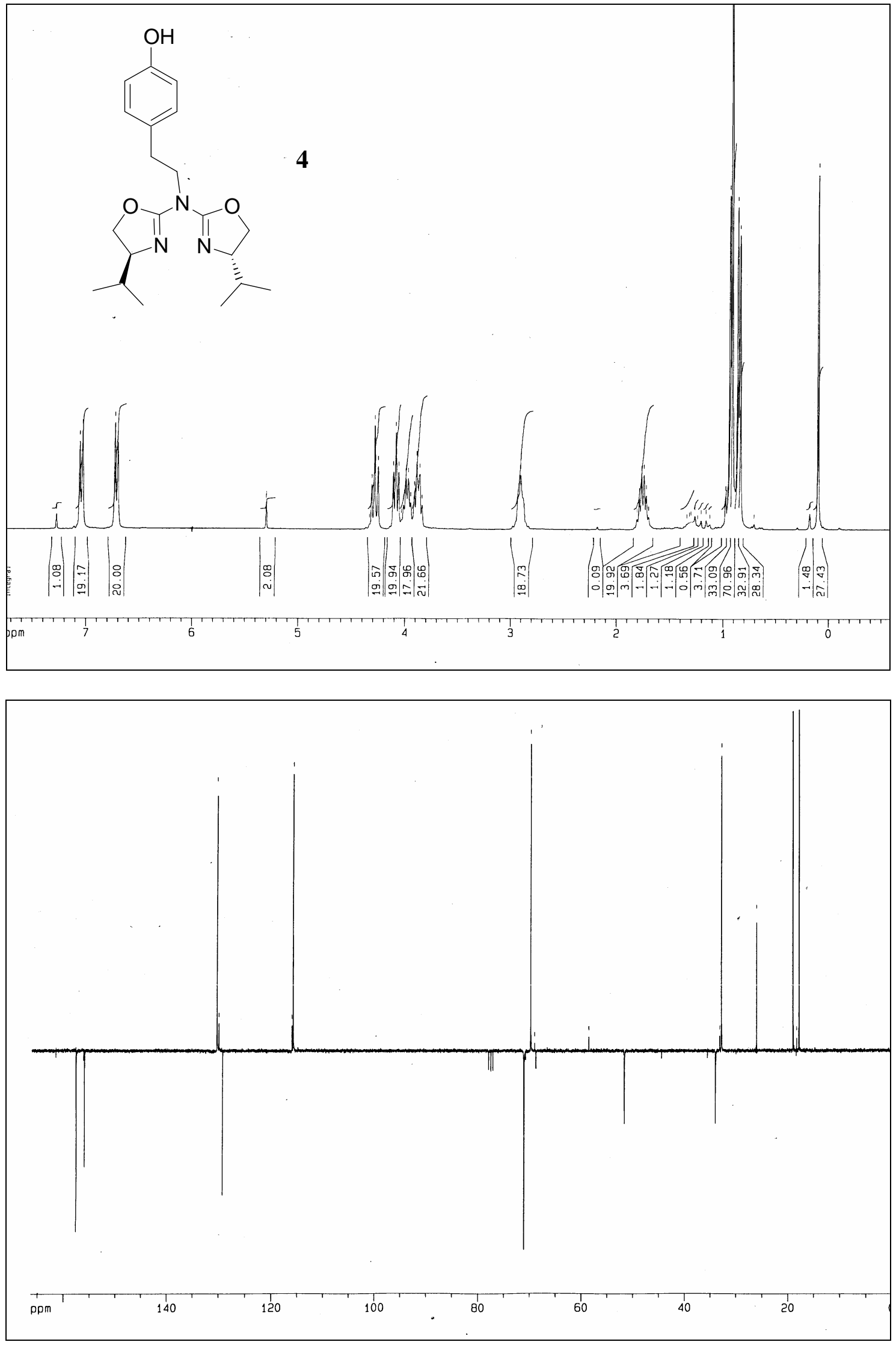
Dodekakis-(4-\{2-[bis-(4-isopropyl-4,5-dihydro-oxazol-2-yl)-amino]-ethyl\}phenoxy)-cyclotriphosphazene $\left(G_{1} \bullet 4\right)$ :
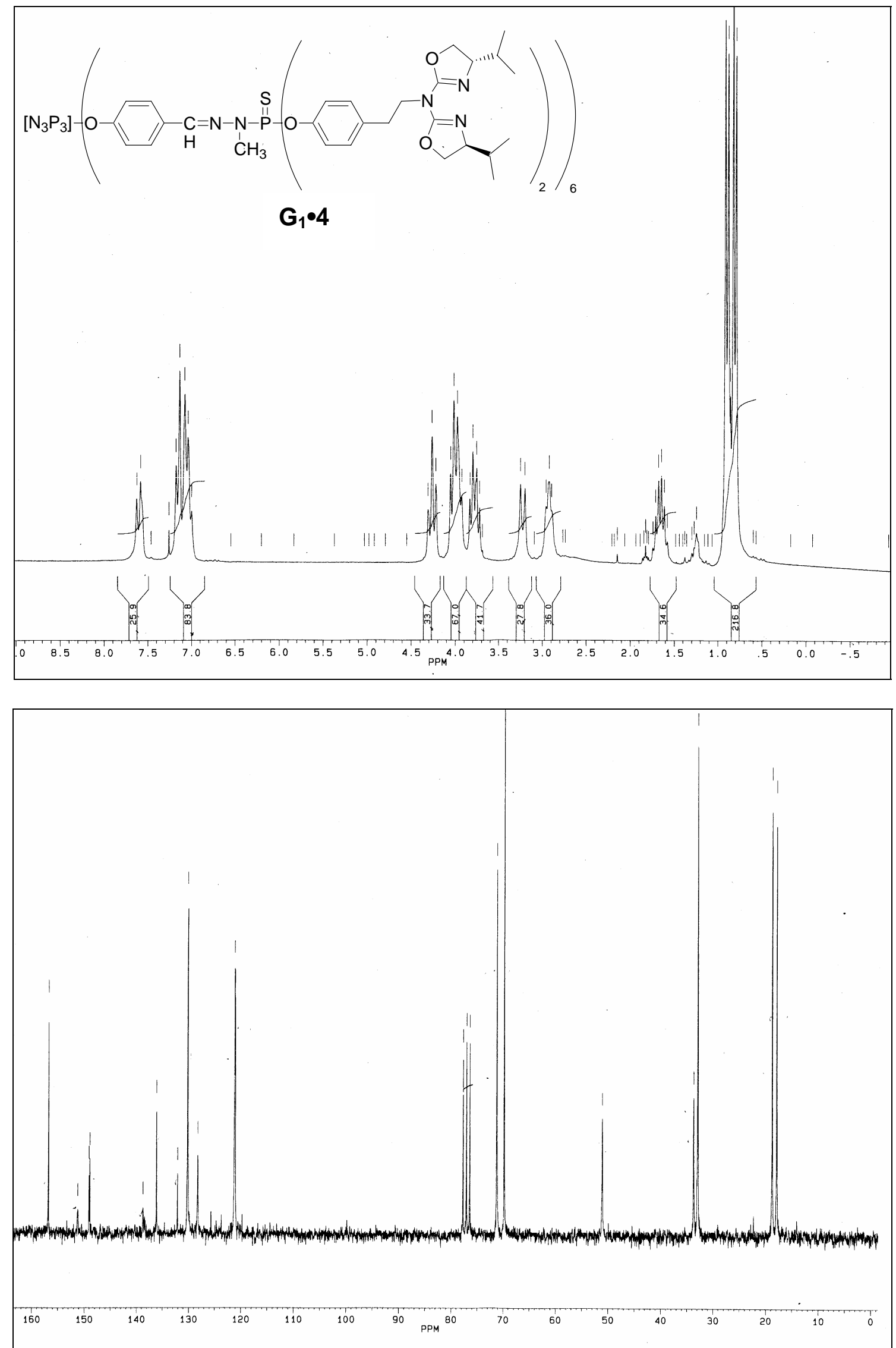
Eikosa-tetrakis-(4-\{2-[bis-(4-isopropyl-4,5-dihydro-oxazol-2-yl)-amino]-ethyl\}phenoxy)-cyclotriphosphazene $\left(G_{2} \bullet 4\right)$ :
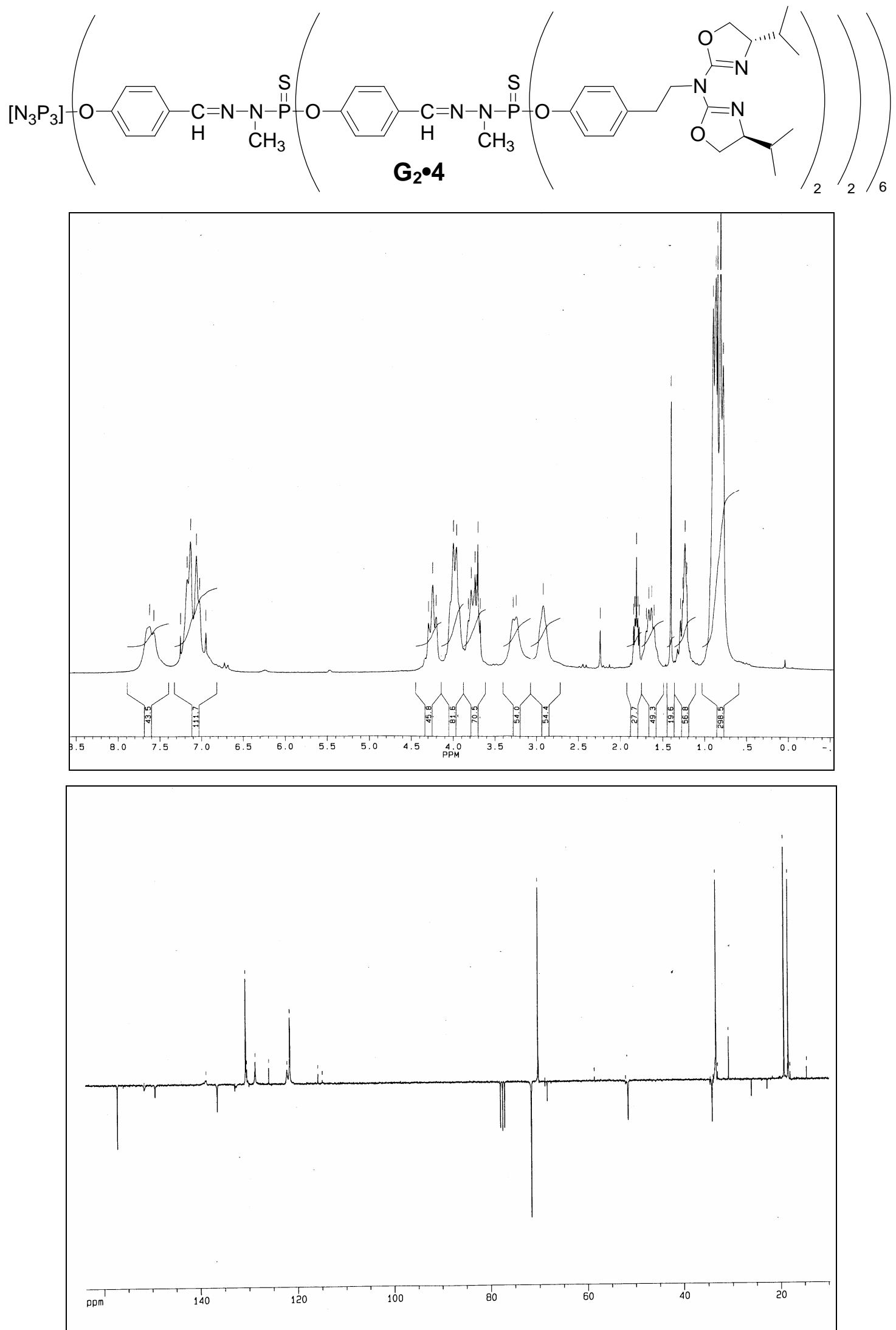
Tessaraconta-oktakis-(4-\{2-[bis-(4-isopropyl-4,5-dihydro-oxazol-2-yl)-amino]ethyl\}-phenoxy)-cyclotriphosphazene $\left(\mathrm{G}_{3} \bullet 4\right)$ :
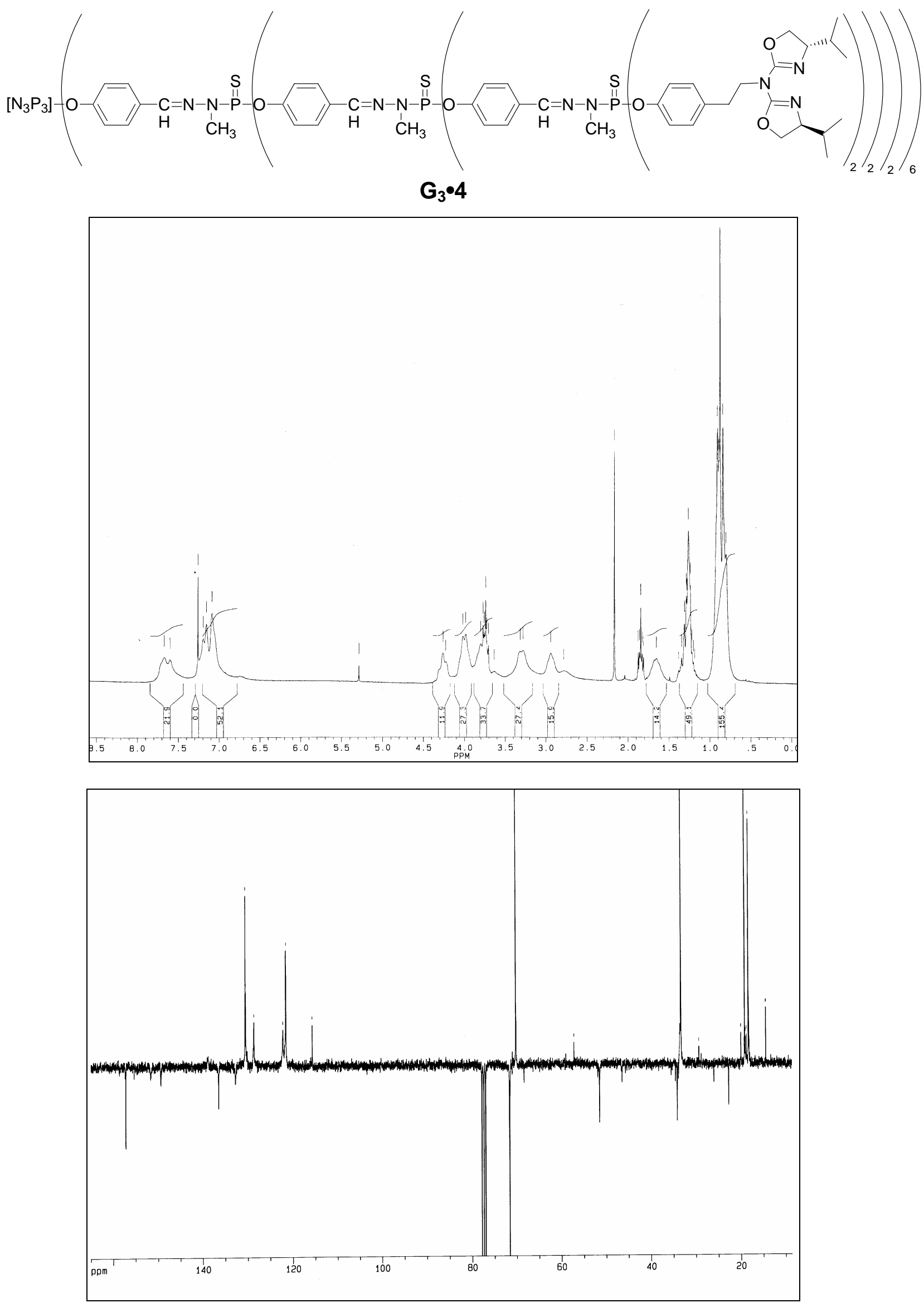


\section{Gc1-Azide $\left(G_{1}\right)$ :}
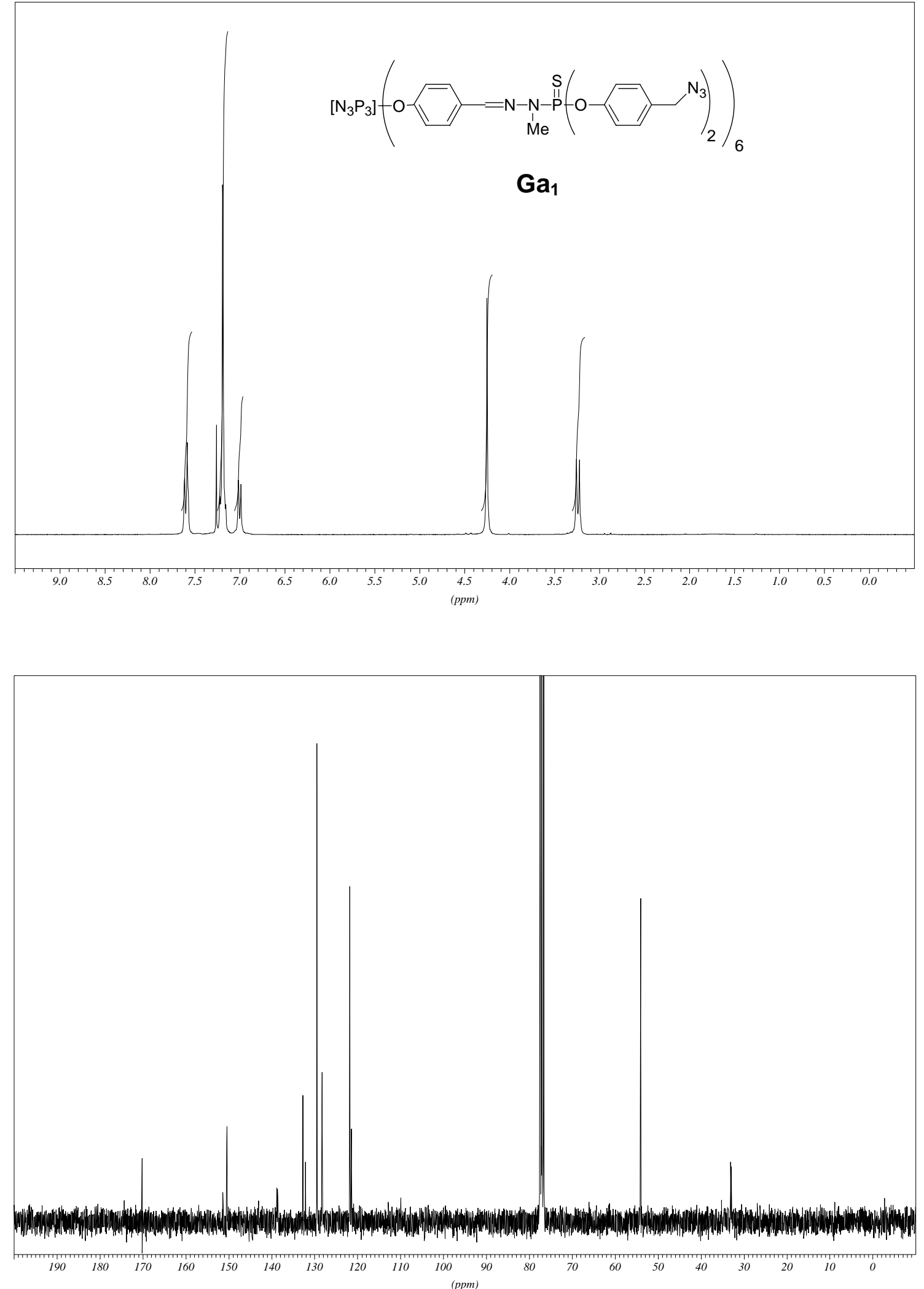

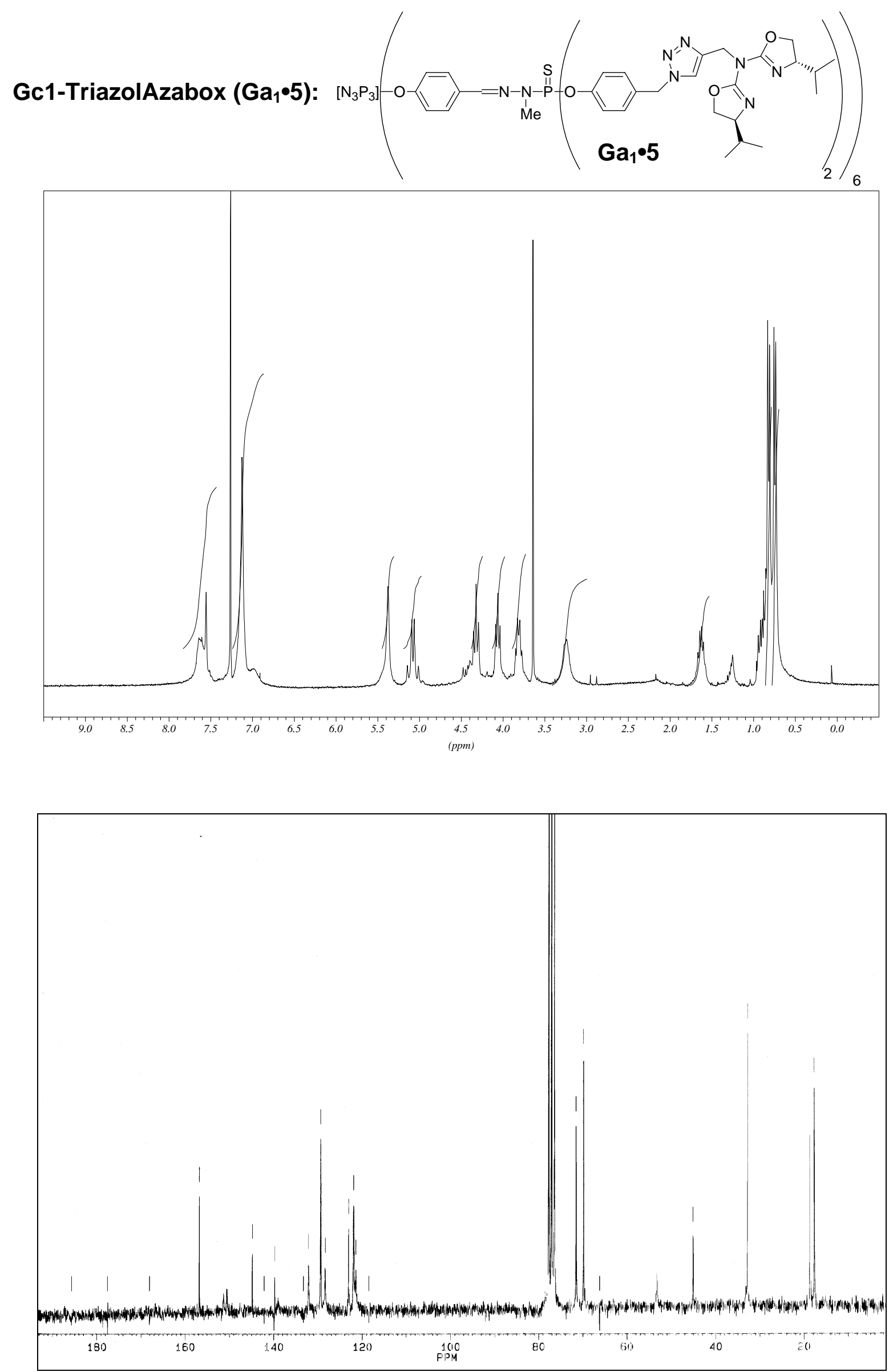
Gc2-Azide $\left(\mathrm{Ga}_{2}\right)$ :

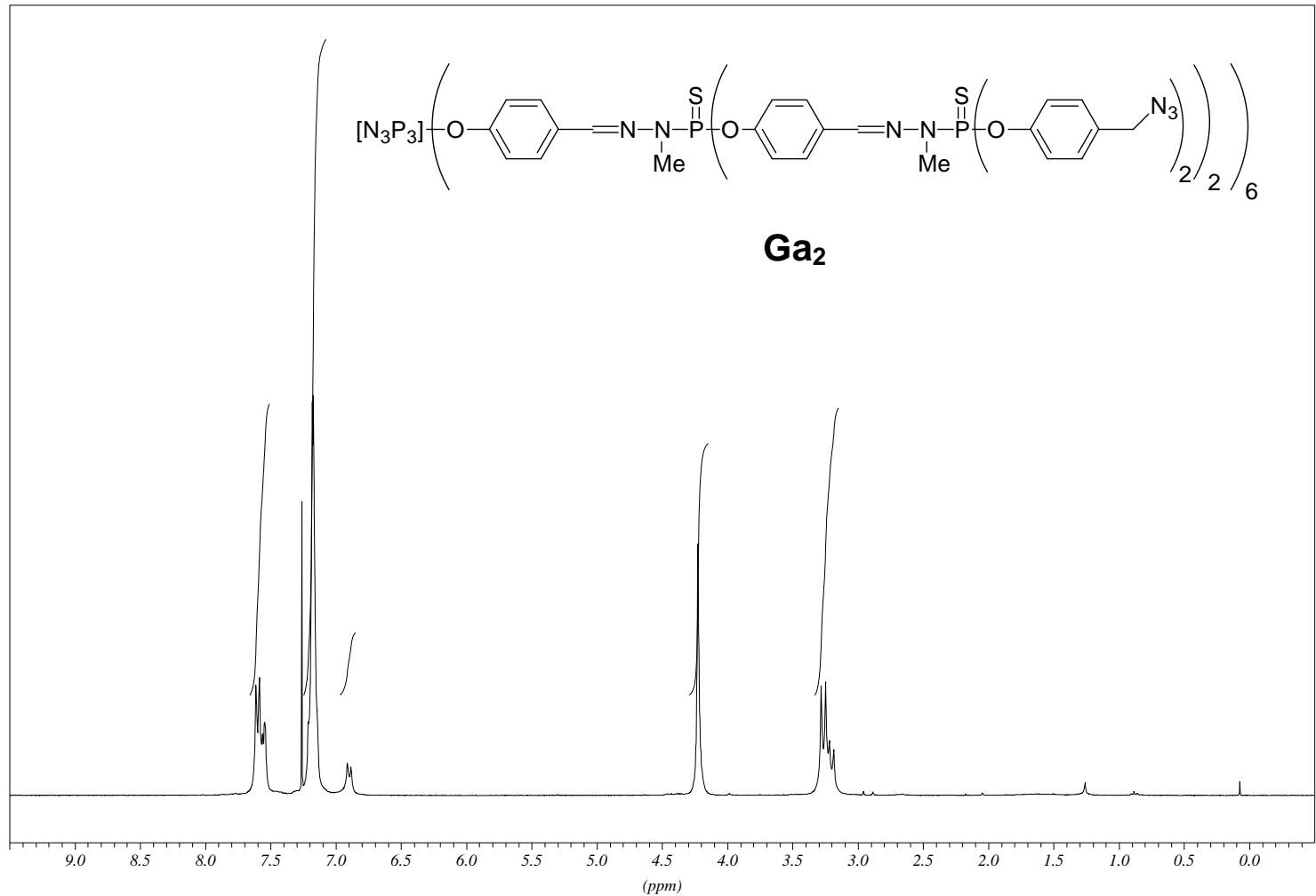

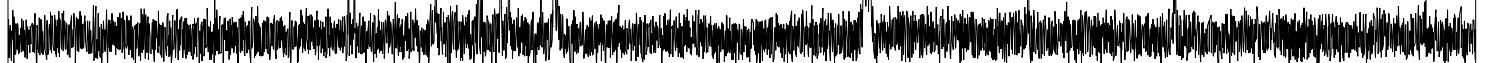

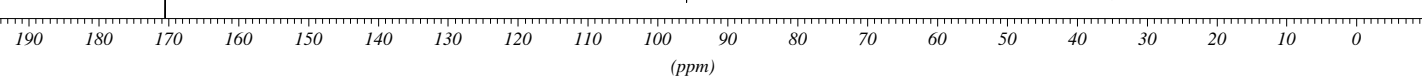



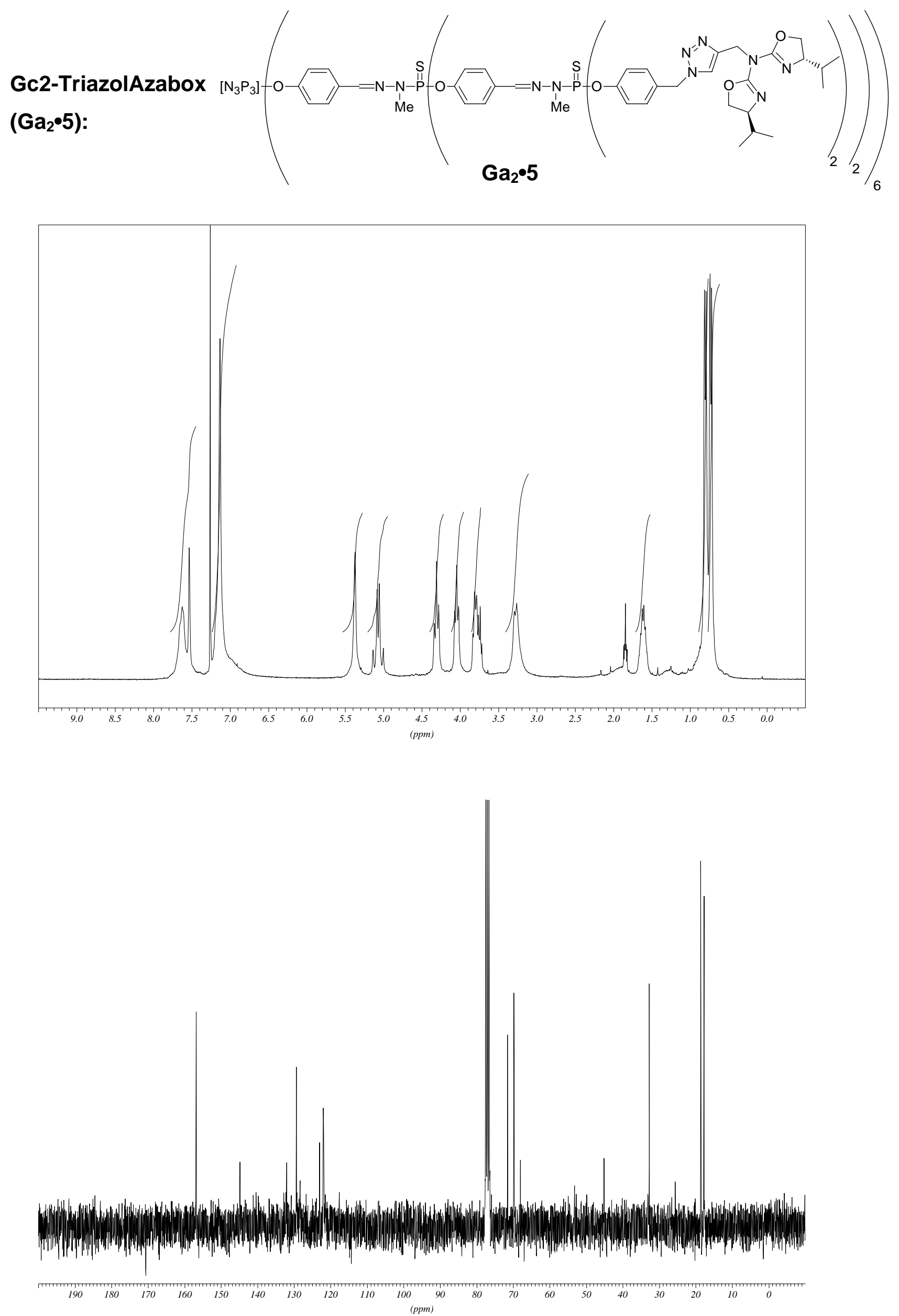
Gc3-Azide $\left(\mathrm{Ga}_{3}\right)$ :
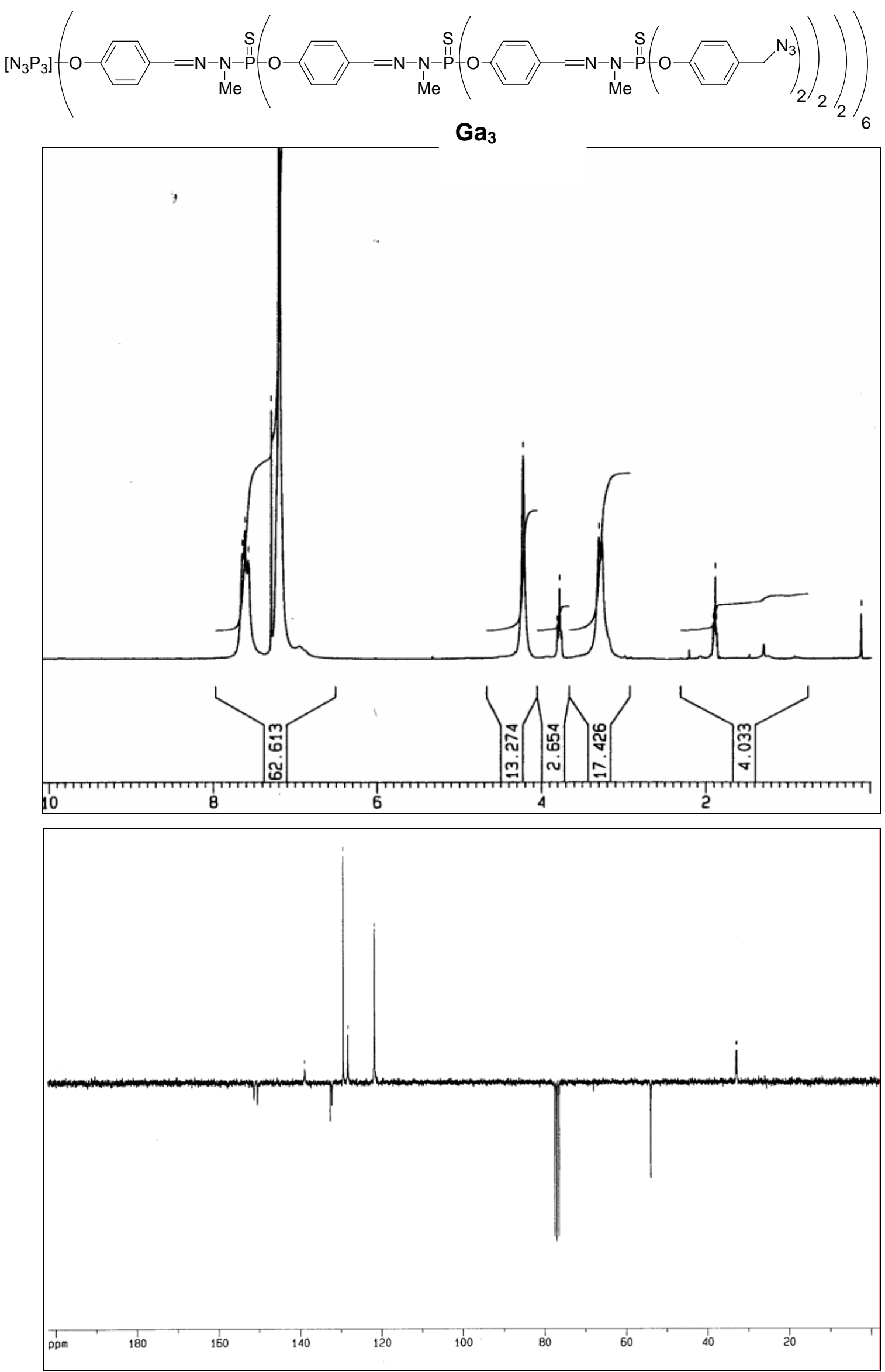
Gc3-TriazolAzabox $\left(\mathrm{Ga}_{3} \cdot 5\right)$ :
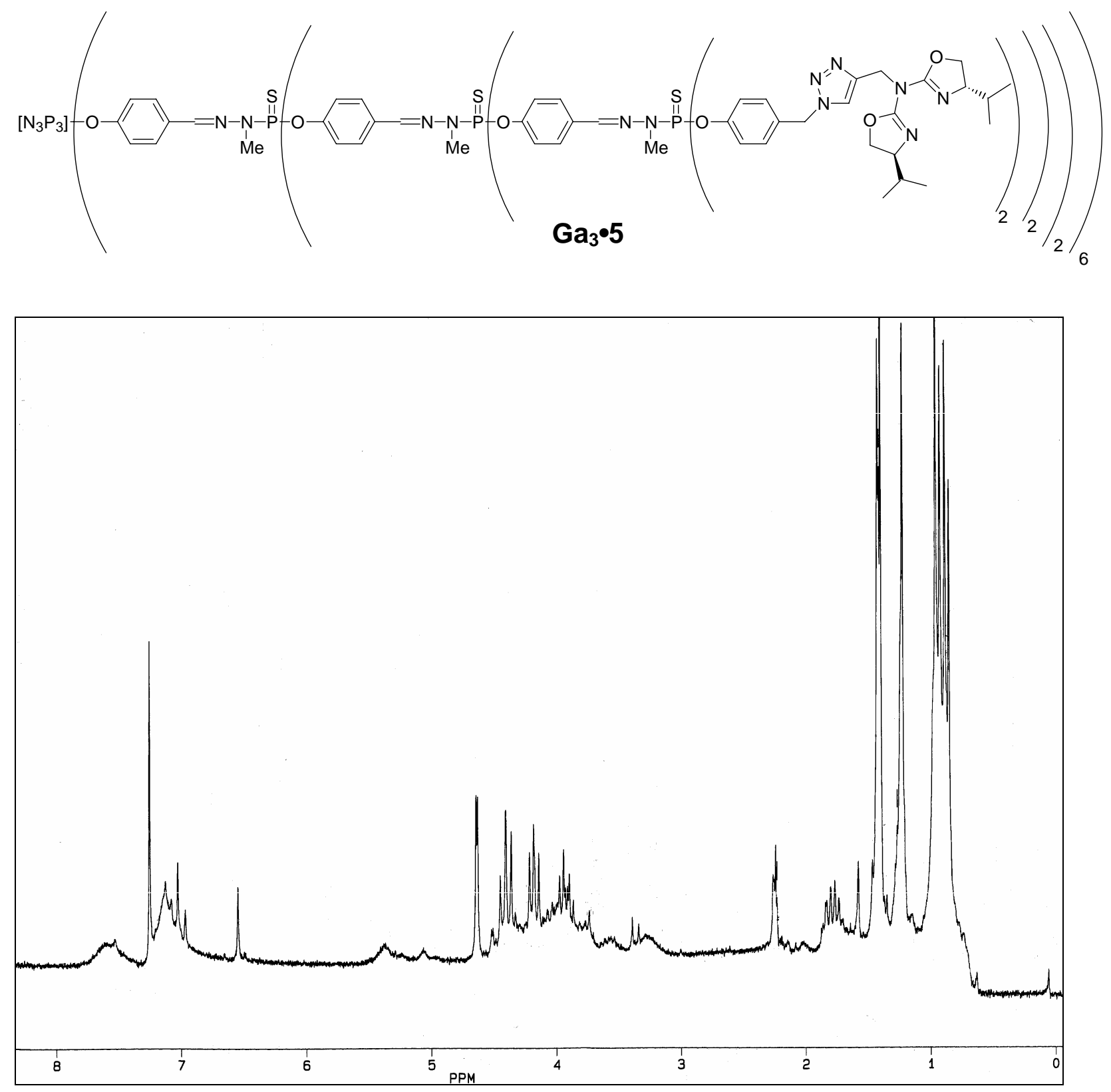